\title{
31. PLAGIOCLASE ZONING IN SELECTED LAVAS FROM HOLES 834B, 839B, AND 841B
}

\author{
Wilfred B. Bryan ${ }^{2}$ and Thomas H. Pearce ${ }^{3}$
}

\begin{abstract}
We use Nomarski differential interference contrast imaging to reveal the wealth of complex detail in plagioclase zoning for selected samples from Sites 834,839 , and 841 . All sites contain some plagioclase with the very complex internal core zoning, convolute zoning, or very fine-scale euhedral oscillatory zoning of the sort generally considered typical of island-arc volcanic rocks. Plagioclase with contrasted zoning styles may coexist within a single lithologic unit or even within a single thin section. Especially notable is the presence of scattered plagioclase phenocrysts with complex zoning throughout Unit 7 in Hole 834B, which in other respects is relatively uniform in composition and appears to have had little or no differential sorting of crystals and liquid.

Although our study is by no means comprehensive, it is sufficient to indicate that magmatic conditions have been variable during crystallization of these rocks, and mixing or at least minor contamination may be required to explain some of the relations observed. By analogy with experimental studies, it is possible that variations in water content, either over time or within different parts of a chamber or conduit system, have contributed to the observed contrasts in zoning.
\end{abstract}

\section{INTRODUCTION}

Plagioclase is generally a stable crystallizing phase in magmas over a wide range of pressures, temperatures, and compositions. Once crystallized, it re-equilibrates very slowly, if at all, to new pressuretemperature (P-T) conditions and usually responds to changes by continued overgrowth of plagioclase with a composition reflecting the new conditions. The successive zones can be used like growth rings in trees to trace the history of magmatic conditions throughout most of the period of magma crystallization. Plagioclase can become unstable as a result of changes in pressure and especially in content of volatile components such as water. This can result in surface dissolution with consequent rounding and resorption of existing crystals, which sometimes produces deep embayments and highly irregular crystal outlines. Subsequent renewal of growth may fill these embayments with plagioclase of different composition, resulting in complex or chaotic zoning patterns. Less complicated surface irregularities appear as "unconformities" in the zoning stratigraphy. Given sufficient time, this new episode of growth may restore normal euhedral crystal outlines, with the chaotic episodes and unconformities preserved internally.

Normal polarized light microscopy can reveal details of plagioclase zoning if zones are substantially broader than the thickness of the thin section, and if the compositional contrast is sufficient. When zoning approaches several tens of microns in breadth, optical resolution begins to suffer, especially if the zone boundary is not perpendicular to the surface of the slide, because the image integrates a finite thickness rather than an infinitely thin surface. Reflected light solves this problem, provided some means exist to differentiate compositions on a surface. Various stains or etches can make this distinction. One of the most sensitive methods, which we have employed, uses fluboric acid, which preferentially dissolves the more calcic plagioclase and leaves the relatively more sodic areas as slightly elevated ridges or plateaus. Nomarski interference contrast, which excels at detailing small differences in relief, is used to image the relief surface produced by the etch (Anderson, 1983) and is capable of resolving detail on the scale of a micron or less. The method does not permit direct determination of

\footnotetext{
${ }^{1}$ Hawkins, J., Parson, L., Allan, J., et al., 1994. Proc. ODP, Sci. Results, 135: College Station, TX (Ocean Drilling Program).

${ }^{2}$ Department of Geology and Geophysics, Woods Hole Oceanographic Institution, Woods Hole, MA 02543, U.S.A.

${ }^{3}$ Department of Geological Sciences, Queen's University, Kingston, Ontario, K7L 3 N6, Canada.
}

composition, but this can be obtained by Laser Interference Microscopy (Pearce, 1984) calibrated by optical or microprobe determinations on selected zones. The Nomarski images of some crystals are supported by electron microprobe analyses made with the automated JEOL microprobe at MIT. These data show the major compositional variations but do not resolve differences between the smallest zone units. These are on the order of 3-4 $\mu \mathrm{m}$ in width, about the size of the probe beam.

Our purpose in this paper is to show the complex zoning patterns present in some selected samples from the Lau Basin and Tonga forearc drill sites. Our terminology, methods, and assumptions in these initial attempts to interpret plagioclase zoning stratigraphy are the same as those recently outlined by Pearce and Kolisnik (1990). Although this is a technique that, to the best of our knowledge, has not previously been applied to volcanic rocks of backarc basins, it is not new. Wiebe (1968) used plagioclase stratigraphy to interpret the magmatic history of a granitic intrusion, and there have been many other such studies, commonly on intrusive rocks.

Our samples were selected to represent the range of magmatic environments covered during Leg 135 . Site 841 , in the forearc, appears to represent an early stage of the present Tonga island arc; Site 839 in the eastern Lau Basin is still in a backarc environment but geochemically shows some arc affinities; and Site 834 in the western Lau Basin is most "MORB-like" in composition (Parson, Hawkins, Allan, et al., 1992). If these geochemically distinguishable sites also reflect different physical environments of magmatic evolution, this might be recorded in differences in plagioclase zoning patterns.

\section{SITE 841-TONGA FOREARC}

The andesite sills from the lower Miocene section of Hole 841B provide an opportunity to examine some of the oldest magmatic activity recorded in the Tonga Arc. We have selected two moderately plagioclase-phyric samples; in each we have selected one well-developed plagioclase phenocryst for illustration and discussion. Plate 1, Figure A, shows part of a euhedral phenocryst with a euhedral core (just visible along the lower edge of the photo) and a broad internal region of "hieroglyphic" zoning, which we interpret as a result of reaction. Individual domains of this reaction zone tend toward a rectangular shape and are on the order of $30-80 \mu \mathrm{m}$ wide. This is a common feature of terrestrial arc lavas and probably reflects a period of prolonged instability during which the dissolution of more sodic plagioclase alternated with the precipitation of more calcic plagioclase. In the most extreme cases, liquid trapped in the reaction zone forms the "fritted" zones common in calc- 
alkaline plagioclase phenocrysts, and which has been reproduced in laboratory experiments by Tsuchiyama (1985).

Figure B of Plate 1 shows a narrow extension of a larger phenocryst. It contrasts with the crystal in Plate 1, Figure A, in that it shows complex curved zoning that nevertheless does not become cross-cutting, as the continuity of individual zones can be followed like topographic contours. This is similar to the convolute zoning described by Blackerby (1968). The convolute zoning is deposited on a euhedral substrate, and the last euhedral zone represents the point at which the interface became unstable and changed from planar to curvilinear. The zoning is generally conformable with only minor suggestions of rounding at some corners. Note that there is a tendency to alternate sharp and rounded vertices, a feature noted by one of us in basaltic andesite from Mount St. Helens (T.H. Pearce, unpubl. data). Note also how the prominent inner embayment ( $\mathrm{E}$ in the figure) gives way to euhedral overgrowth; there is then a return to a hint of instability $\left(E^{\prime}\right)$, followed by more euhedral overgrowth. Another major embayment ( $\left.E^{\prime \prime}\right)$ ultimately is enclosed by a final, outer euhedral rim. As illustrated in this crystal, a given face may carry an apparent "memory" of the innermost anomalous growth event and is later prone to a repetition of that event. This "memory" might be in the form of submicroscopic dislocations that propagate through apparently perfect euhedral faces. Such dislocations may always be more subject to reaction or dissolution than the adjacent normal areas.

Microprobe traverses across the first crystal (Plate 1, Fig. A) define the major compositional variations from core to outer rim. Traverse 1 used hand-selected point analyses to define the major variations from core to rim, whereas traverse 2 was designed to provide more detail in the region of "hieroglyphic" zoning. Individual point analyses are given in Table 1, and schematic profiles along each traverse are given in Figures 1 and 2. A slight tendency is present in both profiles to an increase in $\mathrm{MgO}$ and $\mathrm{Mg} \#$ in the central part of each profile, but overall little systematic variation exists within the chaotic zone. Plate 2 shows an enlarged view of the plagioclase in Plate 1, Figure A, with the principal stratigraphic zones labeled. The outermost surface (A) is scalloped, giving a highly variable thickness to Zone A-B. This averages about $30 \mu \mathrm{m}$ in thickness and is smooth with no dislocations. The composition is about $\mathrm{An}_{75}$. Zone $\mathrm{B}-\mathrm{C}$ is $30-40 \mu \mathrm{m}$ thick and is character- ized by concentric, lobate units separated by steplike dislocations about $25 \mu \mathrm{m}$ apart. Compositional variation is small, averaging about $\mathrm{An}_{79}$. This may be a zone of rapid growth, a precursor to the final quenching event marked by Zone A-B. Point C marks a euhedral interface and an abrupt increase in anorthite content (to $\mathrm{An}_{88-89}$ ). Zone $\mathrm{C}-\mathrm{D}$ is about 45 $\mu \mathrm{m}$ wide and varies little in composition in spite of some 12 thin euhedral zones, which average about $4 \mu \mathrm{m}$ thick. Several prominent dislocations extend through this zone at the upper right. At the far left, an apparent angular unconformity along boundary $\mathrm{C}$ actually may be a sector zone boundary where the zones change direction abruptly, and where Zone C-D becomes much narrower in the other sector. Point D marks the innermost limit of the region of euhedral growth, but it is not a significant composition boundary. Thus, Zone D-E represents a period when a composition of about $\mathrm{An}_{88}$ became stable, but it required about ten oscillations of growth (Zone D-E) to convert the underlying irregular substrate to a euhedral surface. Point E marks the outer limit (reaction surface) of the original crystal, which had been partly resorbed and deeply corroded by reaction with the melt. The reaction zone varies from $\mathrm{An}_{90}$ to $\mathrm{An}_{94}$. Point $\mathrm{F}$ marks the inner limit of the reaction zone and is the euhedral boundary of the inner core. The core shows faint traces of irregular zoning, but it appears to vary little from the analyzed points, which average about $\mathrm{An}_{92}$.

In conclusion, the plagioclases in these sills show patterns similar to those in other circum-Pacific arcs and volcanic belts that we have examined; these include the Trans-Mexican belt (Popocatepetl), the High Cascades (Mount St. Helens), and also the Lesser Antilles (St. Kitts). Complexity in the plagioclase zoning usually is accompanied by other evidence that suggests mixing. Although some mixing may have occurred here, it is difficult to evaluate in an isolated sill. Though convective mixing is an accepted way to change plagioclase compositions, fluctuations in water pressure without any mixing might be equally effective. Experimental data bearing on this point are given by Gaetani et al. (this volume).

\section{HOLE 834B-WESTERN LAU BASIN}

Site 834 samples are mostly sparsely phyric to aphyric; a notable exception is Unit 7 of Hole $834 \mathrm{~B}$, which is characterized by plagio-

Table 1. Electron microprobe analyses of large-zoned plagioclase phenocryst, Hole 841B, Miocene andesite.

\begin{tabular}{|c|c|c|c|c|c|c|c|c|c|c|c|c|}
\hline Point & $\mathrm{SiO}_{2}$ & $\mathrm{Al}_{2} \mathrm{O}_{3}$ & $\mathrm{FeOT}$ & $\mathrm{MgO}$ & $\mathrm{CaO}$ & $\mathrm{Na}_{2} \mathrm{O}$ & $\mathrm{K}_{2} \mathrm{O}$ & Total & $\mathrm{Ab}$ & An & $x$ & $y$ \\
\hline A & 46.98 & 33.13 & 0.68 & 0.11 & 17.30 & 1.61 & 0.02 & 99.83 & 7.74 & 92.18 & 49.70 & 61.75 \\
\hline B & 47.69 & 32.76 & 0.66 & 0.13 & 16.92 & 1.88 & 0.03 & 100.06 & 9.11 & 90.76 & 49.54 & 61.62 \\
\hline C & 47.81 & 32.73 & 0.65 & 0.12 & 17.04 & 1.88 & 0.02 & 100.25 & 9.06 & 90.84 & 49.51 & 61.66 \\
\hline D & 46.35 & 33.86 & 0.70 & 0.09 & 17.75 & 1.34 & 0.03 & 100.12 & 6.38 & 93.51 & 49.41 & 61.67 \\
\hline E & 46.91 & 33.48 & 0.59 & 0.12 & 17.38 & 1.54 & 0.04 & 100.06 & 7.41 & 92.44 & 49.40 & 61.70 \\
\hline $\mathrm{F}$ & 48.90 & 31.75 & 0.71 & 0.14 & 15.90 & 2.50 & 0.04 & 99.95 & 12.45 & 87.38 & 49.39 & 61.68 \\
\hline G & 48.22 & 32.46 & 0.65 & 0.16 & 16.14 & 2.16 & 0.04 & 99.83 & 10.79 & 89.04 & 49.36 & 61.70 \\
\hline $\mathrm{H}$ & 47.99 & 32.16 & 0.75 & 0.11 & 16.41 & 2.14 & 0.04 & 99.60 & 10.53 & 89.32 & 49.32 & 61.66 \\
\hline I & 47.92 & 32.80 & 0.71 & 0.13 & 16.42 & 2.05 & 0.03 & 100.06 & 10.13 & 89.73 & 49.30 & 61.67 \\
\hline J & 52.14 & 29.86 & 0.73 & 0.12 & 13.30 & 3.77 & 0.10 & 100.04 & 20.35 & 79.26 & 49.28 & 61.65 \\
\hline $\mathrm{K}$ & 53.40 & 28.96 & 0.77 & 0.08 & 12.37 & 4.50 & 0.08 & 100.15 & 24.69 & 75.00 & 49.24 & 61.66 \\
\hline 1 & 47.24 & 33.09 & 0.71 & 0.12 & 17.06 & 1.63 & 0.02 & 99.88 & 7.94 & 91.98 & 49.70 & 61.82 \\
\hline 2 & 47.10 & 33.34 & 0.68 & 0.10 & 17.09 & 1.69 & 0.03 & 100.04 & 8.22 & 91.65 & 49.68 & 61.76 \\
\hline 3 & 46.39 & 33.73 & 0.69 & 0.10 & 17.73 & 1.29 & 0.02 & 99.94 & 6.16 & 93.75 & 49.68 & 61.74 \\
\hline 4 & 48.08 & 32.14 & 0.74 & 0.15 & 16.57 & 2.08 & 0.03 & 99.79 & 10.19 & 89.69 & 49.68 & 61.72 \\
\hline 5 & 48.19 & 32.51 & 0.66 & 0.14 & 16.39 & 2.04 & 0.03 & 99.96 & 10.12 & 89.75 & 49.68 & 61.72 \\
\hline 6 & 47.49 & 32.68 & 0.76 & 0.12 & 16.79 & 1.88 & 0.03 & 99.76 & 9.18 & 90.70 & 49.68 & 61.71 \\
\hline 7 & 47.56 & 32.84 & 0.75 & 0.13 & 16.88 & 1.79 & 0.04 & 99.99 & 8.74 & 91.11 & 49.68 & 61.68 \\
\hline 8 & 47.74 & 32.35 & 0.63 & 0.14 & 16.81 & 2.07 & 0.04 & 99.79 & 10.00 & 89.83 & 49.67 & 61.66 \\
\hline 9 & 47.48 & 32.60 & 0.65 & 0.13 & 16.68 & 1.94 & 0.03 & 99.51 & 9.52 & 90.35 & 49.67 & 61.64 \\
\hline 10 & 47.35 & 33.10 & 0.63 & 0.14 & 17.11 & 1.83 & 0.04 & 100.20 & 8.82 & 91.04 & 49.67 & 61.62 \\
\hline 11 & 46.97 & 33.20 & 0.68 & 0.13 & 17.26 & 1.63 & 0.03 & 99.89 & 7.85 & 92.04 & 49.67 & 61.60 \\
\hline 12 & 47.34 & 32.62 & 0.74 & 0.14 & 16.76 & 1.90 & 0.03 & 99.53 & 9.29 & 90.59 & 49.67 & 61.58 \\
\hline 13 & 47.09 & 32.98 & 0.72 & 0.11 & 17.00 & 1.69 & 0.03 & 99.62 & 8.22 & 91.67 & 49.66 & 61.55 \\
\hline 14 & 46.65 & 33.28 & 0.67 & 0.11 & 17.35 & 1.47 & 0.02 & 99.55 & 7.13 & 92.76 & 49.66 & 61.53 \\
\hline 15 & 47.68 & 32.44 & 0.74 & 0.09 & 16.64 & 1.97 & 0.04 & 99.61 & 9.66 & 90.17 & 49.66 & 61.51 \\
\hline 16 & 46.88 & 33.45 & 0.66 & 0.11 & 17.55 & 1.52 & 0.03 & 100.20 & 7.27 & 92.59 & 49.66 & 61.49 \\
\hline 17 & 46.95 & 33.71 & 0.70 & 0.12 & 17.25 & 1.52 & 0.02 & 100.27 & 7.39 & 92.51 & 49.62 & 61.45 \\
\hline
\end{tabular}

Notes: Data determined on Sample 135-841B-18R-2, 14-18 cm, Subunit 1A. Lettered points are from traverse 1, numbered points are from traverse 2. See Plate 1 for traverse map. All oxide data in weight percent; $\mathrm{Ab}$ and $\mathrm{An}$ in molecular percent. 

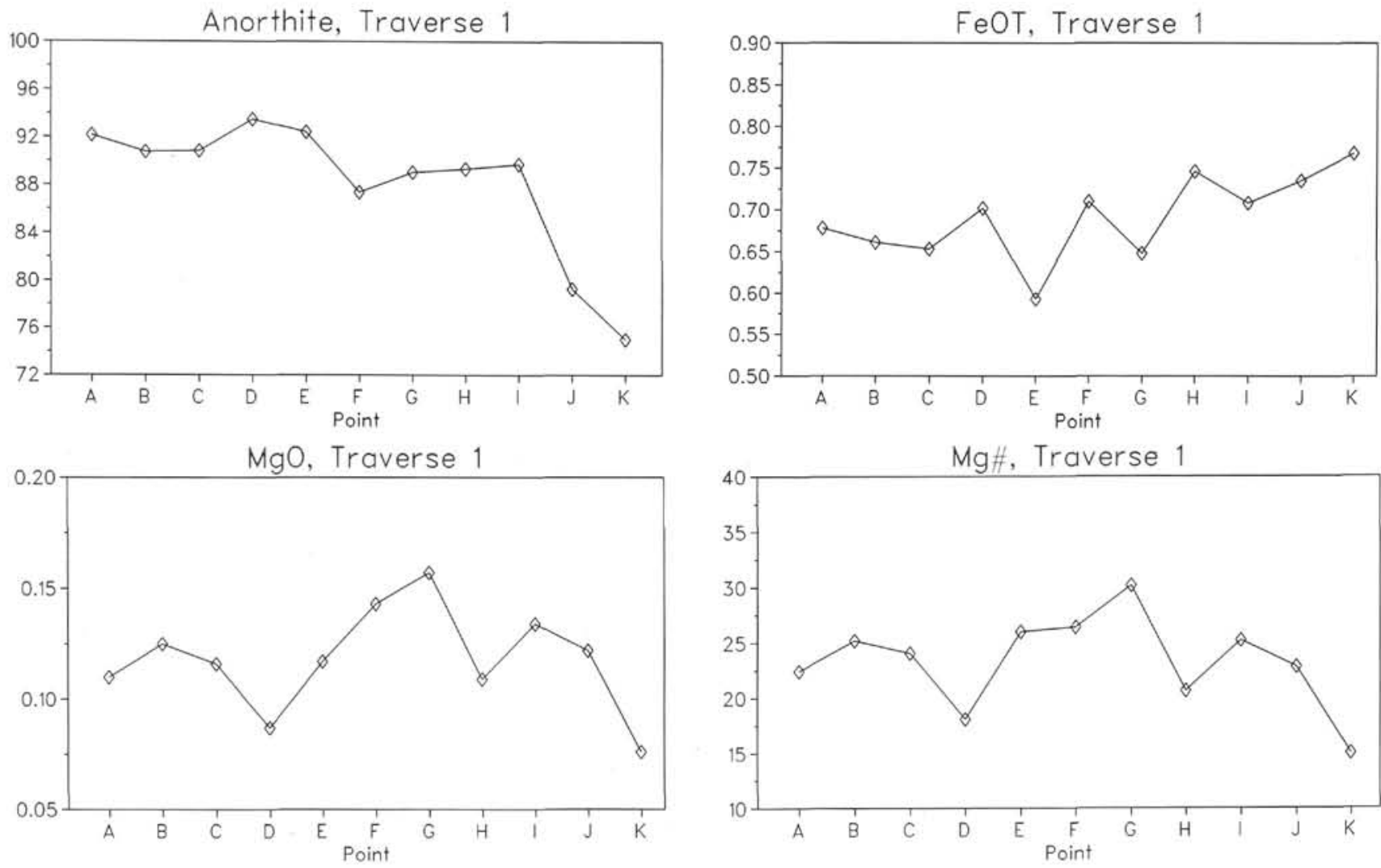

Figure 1. Electron microprobe traverse 1 (diamonds indicate the track in Plate 1, Fig. A); Sample 135-841B-18R-2, 14-18 cm. Points are in order but not to scale. Length of traverse is about $100 \mu \mathrm{m}$.
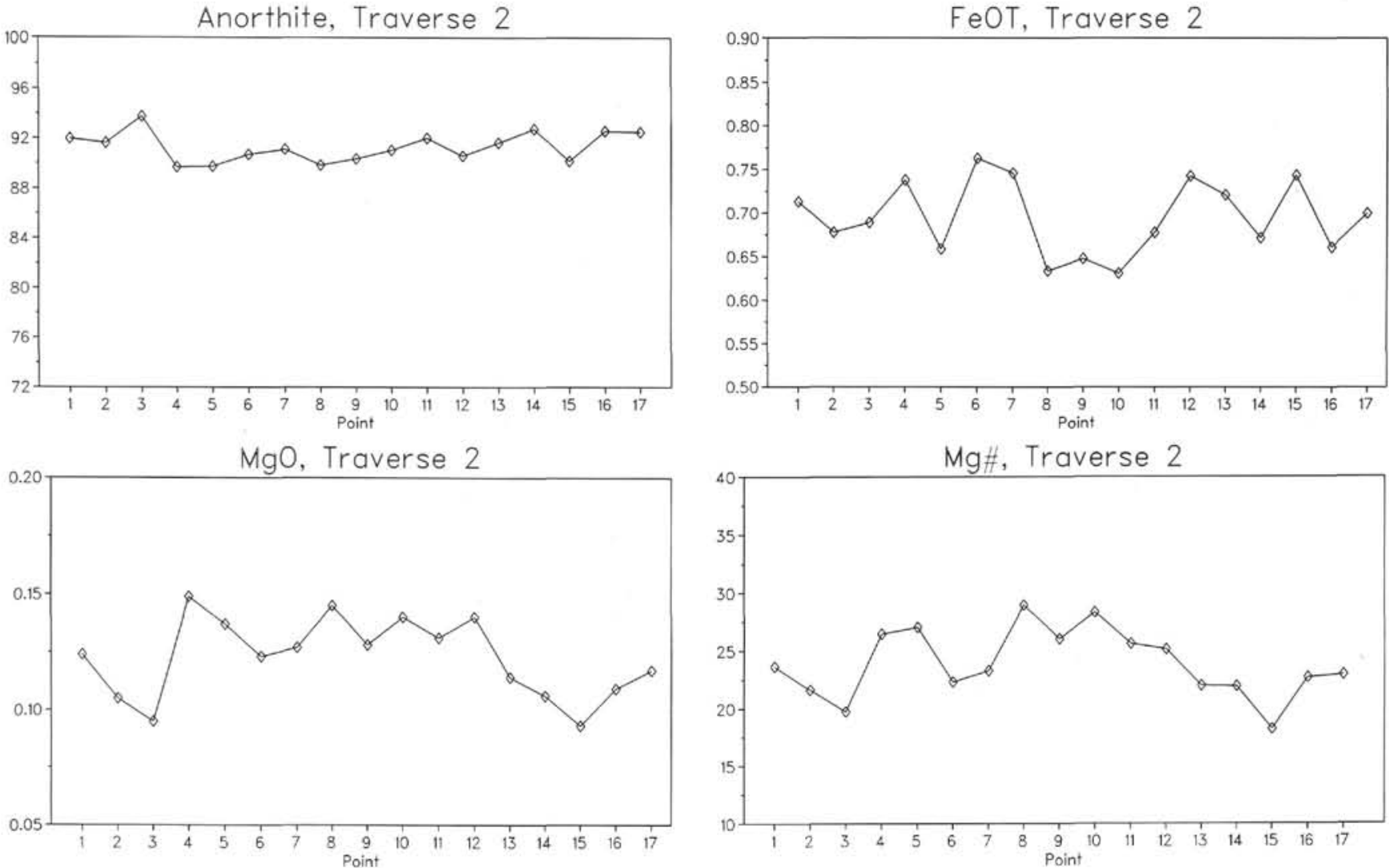

Figure 2. Electron microprobe traverse 2 (squares in Plate 1, Fig. A); Sample 135-841B-25R-4, 10-14 cm. Points are in order but spacing is not to exact scale. Length of traverse is about $95 \mu \mathrm{m}$. 

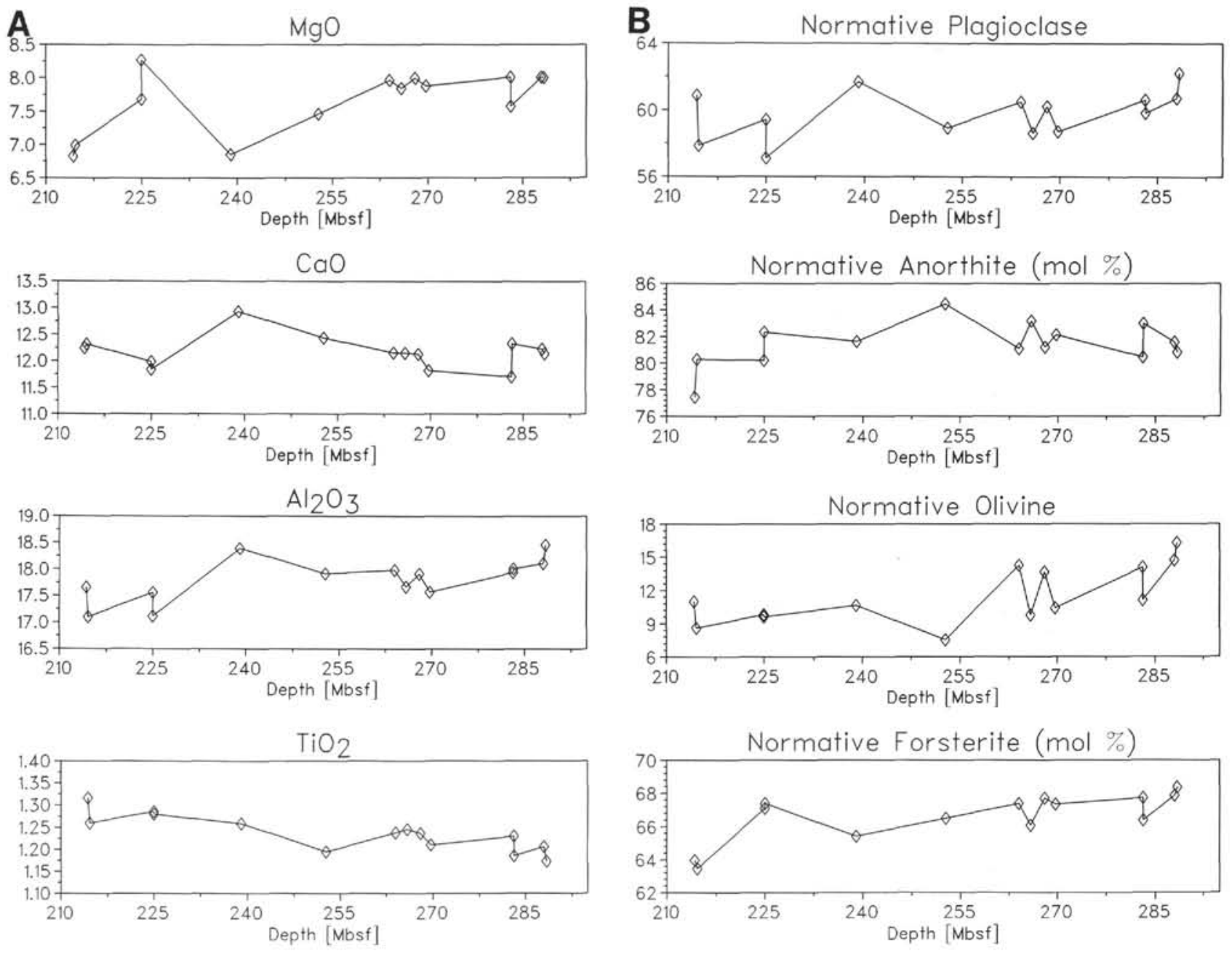

Figure 3. A. Variation of major element oxides with depth in Hole 834B, Unit 7. B. Variation of calculated normative parameters and $\mathrm{Mg} \#$ with depth in Hole 834B, Unit 7. See text for discussion.

clase phenocrysts that constitute $10 \%-15 \%$ of the rock by volume, along with several percent of olivine phenocrysts. We have made a reconnaissance study of Unit 7 that included both Nomarski imaging of plagioclase and microprobe analyses of selected phenocrysts, both plagioclase and olivine (Tables 2,3).

This is the thickest contiguous unit penetrated at Hole 834B, being about $75 \mathrm{~m}$ thick. A small variation is present in the bulk-rock composition of the principal major element oxides (Fig. 3A) and of related normative components (Fig. 3B), which are weakly correlated with depth. Except for the "MgO kick" at about $225 \mathrm{mbsf}$, this involves a subtle change from "more primitive" at the base to "more evolved" at the top of the unit. In spite of the phyric nature of Unit 7, the overall bulk composition does not differ appreciably from Unit 5 , which is aphyric to sparsely phyric. We do not find any obvious correlation between bulk composition and the modal abundance of phenocrysts observed in thin section; similarly, preliminary calculations of phenocryst abundances at varying depths, using bulk-rock and microprobe analyses, do not show any systematic variation in phase abundances with depth. Also, glass compositions from Unit 7 (Hergt and Nilsson, this volume) are similar to the bulk-rock analyses and do not vary appreciably from the top of the unit to the bottom, although they do differ consistently from the bulk rocks in being slightly lower in $\mathrm{Al}_{2} \mathrm{O}_{3}$ and $\mathrm{CaO}$, and higher in $\mathrm{SiO}_{2}$ and $\mathrm{TiO}_{2}$. These differences are those expected because of the content of plagioclase phenocrysts in the bulk rock. We tentatively conclude that the bulk-rock compositions repre- sent magmatic liquids that crystallized varying amounts of plagioclase and olivine phenocrysts in situ, with little differential sorting of crystals and liquid before eruption.

The chemical similarity within Unit 7 is also supported by the phenocryst assemblages (plagioclase dominant with minor olivine), and by the similarity in composition of typical phenocrysts. Olivine varies little $\left(\mathrm{Fo}_{86-88}\right)$ throughout the unit and is not appreciably zoned. Plagioclase consistently has calcic cores zoned to variably more sodic compositions in narrow rims, as shown in Figures 4 and 5. Minor elements ( $\mathrm{Fe}$ and $\mathrm{Mg}$ ) generally increase with decreasing anorthite content, but some irregularity exists in the $\mathrm{Mg} / \mathrm{Fe}$ ratios; some grains show an increase followed by a decrease, similar to the effect observed in the Site 841 plagioclase. As shown below, the presence of more complexly zoned plagioclase, possibly xenocrystal, hints at an earlier, more complex history for this magma. Table 4 gives the results of electron microbe analysis of spinel.

We will not discuss zoning variations in detail here, but we will illustrate the variety of crystal forms that appear in Unit 7. The most common phenocryst type is illustrated in Figures $6 \mathrm{~A}$ and $6 \mathrm{~B}$, from the upper and lower parts of Unit 7, respectively. These show faint, diffuse zoning within a broad, calcic central core, with a relatively narrow oscillatory-zoned rim. As indicated in the profiles (Figs. 4-5), these cores average around $\mathrm{An}_{92}$, whereas the rims vary from $\mathrm{An}_{65}$ to $\mathrm{An}_{85}$. However, a few phenocrysts (or xenocrysts?) have more complex cores (Fig. 7) with "hieroglyphic" zoning as discussed with the Site 841 
Table 2. Electron microprobe analyses of plagioclase, Hole 834B, Unit 7.

\begin{tabular}{|c|c|c|c|c|c|c|c|c|c|c|}
\hline Mineral & Grain & $\mathrm{SiO}_{2}$ & $\mathrm{Al}_{2} \mathrm{O}_{3}$ & FeOT & $\mathrm{MgO}$ & $\mathrm{CaO}$ & $\mathrm{Na}_{2} \mathrm{O}$ & $\mathrm{K}_{2} \mathrm{O}$ & Total & An \\
\hline \multicolumn{11}{|c|}{ Sample 135-834B-25R-1, 28-32 cm: } \\
\hline $\begin{array}{l}\text { Plag } 1 \\
\text { Plag 1 } \\
\text { Plag 1 } \\
\text { Plag 1 } \\
\text { Plag 1 } \\
\text { Average }\end{array}$ & $\begin{array}{l}\mathrm{C} \\
\mathrm{C} \\
\mathrm{C} \\
\mathrm{C} \\
\mathrm{C}\end{array}$ & $\begin{array}{l}46.63 \\
47.69 \\
47.00 \\
47.73 \\
53.33 \\
48.48\end{array}$ & $\begin{array}{l}32.86 \\
32.97 \\
33.44 \\
33.82 \\
29.49 \\
32.52\end{array}$ & $\begin{array}{l}0.32 \\
0.38 \\
0.34 \\
0.39 \\
0.60 \\
0.41\end{array}$ & $\begin{array}{l}0.17 \\
0.22 \\
0.18 \\
0.19 \\
0.31 \\
0.21\end{array}$ & $\begin{array}{l}16.51 \\
16.50 \\
17.18 \\
17.09 \\
12.84 \\
16.02\end{array}$ & $\begin{array}{l}1.66 \\
2.05 \\
1.65 \\
1.76 \\
4.04 \\
2.23\end{array}$ & $\begin{array}{l}0 \\
0 \\
0 \\
0 \\
0 \\
0\end{array}$ & $\begin{array}{r}98.15 \\
99.80 \\
99.79 \\
100.97 \\
100.61 \\
99.87\end{array}$ & $\begin{array}{l}91.62 \\
89.88 \\
91.97 \\
91.46 \\
77.82 \\
88.55\end{array}$ \\
\hline $\begin{array}{l}\text { Plag } 2 \\
\text { Plag 2 } \\
\text { Plag 2 } \\
\text { Plag 2 } \\
\text { Plag 2 } \\
\text { Plag 2 } \\
\text { Plag 2 } \\
\text { Plag 2 } \\
\text { Plag 2 } \\
\text { Plag 2 } \\
\text { Average }\end{array}$ & $\begin{array}{l}\mathbf{J} \\
\mathbf{J} \\
\mathbf{J} \\
\mathbf{J} \\
\mathbf{J} \\
\mathbf{J} \\
\mathbf{J} \\
\mathbf{J} \\
\mathbf{J} \\
\mathbf{J}\end{array}$ & $\begin{array}{l}47.08 \\
47.27 \\
47.17 \\
47.54 \\
47.20 \\
47.09 \\
48.07 \\
48.38 \\
47.88 \\
53.17 \\
48.08\end{array}$ & $\begin{array}{l}33.56 \\
33.61 \\
33.55 \\
33.38 \\
33.59 \\
33.04 \\
33.01 \\
32.99 \\
32.81 \\
29.01 \\
32.86\end{array}$ & $\begin{array}{l}0.32 \\
0.33 \\
0.30 \\
0.41 \\
0.35 \\
0.32 \\
0.37 \\
0.34 \\
0.37 \\
0.80 \\
0.39\end{array}$ & $\begin{array}{l}0.17 \\
0.20 \\
0.19 \\
0.18 \\
0.19 \\
0.19 \\
0.22 \\
0.22 \\
0.20 \\
0.48 \\
0.22\end{array}$ & $\begin{array}{l}17.32 \\
17.33 \\
17.44 \\
17.09 \\
17.28 \\
16.68 \\
16.73 \\
16.56 \\
16.35 \\
13.08 \\
16.59\end{array}$ & $\begin{array}{l}1.57 \\
1.58 \\
1.61 \\
1.73 \\
1.79 \\
1.72 \\
1.94 \\
2.03 \\
2.06 \\
4.09 \\
2.01\end{array}$ & $\begin{array}{l}0 \\
0 \\
0 \\
0 \\
0 \\
0 \\
0 \\
0 \\
0 \\
0.02 \\
0\end{array}$ & $\begin{array}{r}100.03 \\
100.32 \\
100.26 \\
100.33 \\
100.40 \\
99.04 \\
100.34 \\
100.53 \\
99.67 \\
100.64 \\
100.16\end{array}$ & $\begin{array}{l}92.37 \\
92.35 \\
92.27 \\
91.59 \\
91.42 \\
91.44 \\
90.48 \\
90.00 \\
89.75 \\
77.85 \\
89.95\end{array}$ \\
\hline \multicolumn{11}{|c|}{ Sample 135-834B-25R-1, $106-110 \mathrm{~cm}$ : } \\
\hline $\begin{array}{l}\text { Plag 1 } \\
\text { Plag 1 } \\
\text { Plag 1 } \\
\text { Plag 1 } \\
\text { Plag 1 } \\
\text { Plag 1 } \\
\text { Plag 1 } \\
\text { Average }\end{array}$ & $\begin{array}{l}\mathrm{G} \\
\mathrm{G} \\
\mathrm{G} \\
\mathrm{G} \\
\mathrm{G} \\
\mathrm{G} \\
\mathrm{G}\end{array}$ & $\begin{array}{l}47.14 \\
47.42 \\
47.22 \\
46.84 \\
47.84 \\
47.17 \\
52.41 \\
48.01\end{array}$ & $\begin{array}{l}33.67 \\
33.42 \\
33.29 \\
33.73 \\
33.38 \\
33.78 \\
29.93 \\
33.03\end{array}$ & $\begin{array}{l}0.23 \\
0.31 \\
0.29 \\
0.35 \\
0.28 \\
0.30 \\
0.51 \\
0.33\end{array}$ & $\begin{array}{l}0.18 \\
0.18 \\
0.16 \\
0.12 \\
0.18 \\
0.17 \\
0.25 \\
0.18\end{array}$ & $\begin{array}{l}17.43 \\
17.32 \\
17.38 \\
17.60 \\
16.77 \\
17.52 \\
13.40 \\
16.78\end{array}$ & $\begin{array}{l}1.56 \\
1.63 \\
1.60 \\
1.48 \\
1.90 \\
1.65 \\
3.84 \\
1.95\end{array}$ & $\begin{array}{l}0.04 \\
0 \\
0.03 \\
0 \\
0 \\
0 \\
0.02 \\
0.01\end{array}$ & $\begin{array}{r}100.26 \\
100.28 \\
99.97 \\
100.13 \\
100.36 \\
100.59 \\
100.37 \\
100.28\end{array}$ & $\begin{array}{l}92.37 \\
92.14 \\
92.21 \\
92.90 \\
90.68 \\
92.13 \\
79.30 \\
90.25\end{array}$ \\
\hline $\begin{array}{l}\text { Plag } 2 \\
\text { Plag } 2 \\
\text { Plag } 2 \\
\text { Plag } 2 \\
\text { Plag 2 } \\
\text { Plag 2 } \\
\text { Average }\end{array}$ & $\begin{array}{l}\text { B } \\
\text { B } \\
\text { B } \\
\text { B } \\
\text { B } \\
\text { B }\end{array}$ & $\begin{array}{l}47.04 \\
47.19 \\
46.88 \\
47.01 \\
47.08 \\
51.37 \\
47.76\end{array}$ & $\begin{array}{l}32.66 \\
33.05 \\
33.21 \\
32.98 \\
33.05 \\
30.10 \\
32.51\end{array}$ & $\begin{array}{l}0.33 \\
0.32 \\
0.28 \\
0.32 \\
0.31 \\
0.42 \\
0.33\end{array}$ & $\begin{array}{l}0.16 \\
0.16 \\
0.16 \\
0.16 \\
0.16 \\
0.28 \\
0.18\end{array}$ & $\begin{array}{l}17.17 \\
17.03 \\
17.38 \\
17.44 \\
17.11 \\
14.13 \\
16.71\end{array}$ & $\begin{array}{l}1.64 \\
1.72 \\
1.68 \\
1.59 \\
1.85 \\
3.41 \\
1.98\end{array}$ & $\begin{array}{l}0.04 \\
0 \\
0 \\
0.03 \\
0 \\
0.02 \\
0.02\end{array}$ & $\begin{array}{l}99.04 \\
99.47 \\
99.60 \\
99.53 \\
99.57 \\
99.73 \\
99.49\end{array}$ & $\begin{array}{l}91.88 \\
91.61 \\
91.91 \\
92.26 \\
91.04 \\
82.01 \\
90.12\end{array}$ \\
\hline \multicolumn{11}{|c|}{ Sample 135-834B-26R-1, 73-77 cm: } \\
\hline $\begin{array}{l}\text { Plag 1 } \\
\text { Plag 1 } \\
\text { Plag 1 } \\
\text { Plag 1 } \\
\text { Plag 1 } \\
\text { Average }\end{array}$ & $\begin{array}{l}F \\
F \\
F \\
F \\
F\end{array}$ & $\begin{array}{l}47.55 \\
47.50 \\
48.06 \\
50.50 \\
50.78 \\
48.88\end{array}$ & $\begin{array}{l}33.46 \\
33.27 \\
32.96 \\
33.54 \\
31.10 \\
32.87\end{array}$ & $\begin{array}{l}0.27 \\
0.27 \\
0.29 \\
0.31 \\
0.44 \\
0.32\end{array}$ & $\begin{array}{l}0.17 \\
0.20 \\
0.21 \\
0.20 \\
0.24 \\
0.20\end{array}$ & $\begin{array}{l}17.09 \\
16.90 \\
16.70 \\
17.07 \\
14.48 \\
16.44\end{array}$ & $\begin{array}{l}1.72 \\
1.80 \\
2.07 \\
1.70 \\
3.25 \\
2.11\end{array}$ & $\begin{array}{l}0.03 \\
0 \\
0.03 \\
0.03 \\
0.04 \\
0.02\end{array}$ & $\begin{array}{r}100.27 \\
99.94 \\
100.32 \\
103.35 \\
100.34 \\
100.84\end{array}$ & $\begin{array}{l}91.56 \\
91.16 \\
89.81 \\
91.63 \\
82.96 \\
89.43\end{array}$ \\
\hline \multicolumn{11}{|c|}{ Sample 135-834B-33R-2, 43-47 cm: } \\
\hline $\begin{array}{l}\text { Plag 1 } \\
\text { Plag 1 } \\
\text { Plag 1 } \\
\text { Plag 1 } \\
\text { Plag 1 } \\
\text { Average }\end{array}$ & $\begin{array}{l}\mathrm{A} \\
\mathrm{A} \\
\mathrm{A} \\
\mathrm{A} \\
\mathrm{A}\end{array}$ & $\begin{array}{l}47.32 \\
47.28 \\
46.97 \\
47.55 \\
53.72 \\
48.57\end{array}$ & $\begin{array}{l}33.73 \\
33.89 \\
33.68 \\
33.37 \\
29.41 \\
32.82\end{array}$ & $\begin{array}{l}0.35 \\
0.31 \\
0.26 \\
0.33 \\
0.57 \\
0.36\end{array}$ & $\begin{array}{l}0.17 \\
0.20 \\
0.17 \\
0.20 \\
0.27 \\
0.20\end{array}$ & $\begin{array}{l}17.12 \\
17.19 \\
17.24 \\
16.73 \\
12.61 \\
16.18\end{array}$ & $\begin{array}{l}1.67 \\
1.59 \\
1.72 \\
1.86 \\
4.29 \\
2.23\end{array}$ & $\begin{array}{l}0.02 \\
0.03 \\
0.02 \\
0.02 \\
0.05 \\
0.03\end{array}$ & $\begin{array}{l}100.39 \\
100.49 \\
100.06 \\
100.06 \\
100.91 \\
100.38\end{array}$ & $\begin{array}{l}91.83 \\
92.14 \\
91.63 \\
90.75 \\
76.32 \\
88.53\end{array}$ \\
\hline $\begin{array}{l}\text { Plag 2 } \\
\text { Plag 2 } \\
\text { Plag 2 } \\
\text { Plag 2 } \\
\text { Plag 2 } \\
\text { Average }\end{array}$ & $\begin{array}{l}Q \\
Q \\
Q \\
Q \\
Q\end{array}$ & $\begin{array}{l}51.24 \\
50.77 \\
50.80 \\
51.11 \\
50.43 \\
50.87\end{array}$ & $\begin{array}{l}30.81 \\
31.21 \\
31.40 \\
31.36 \\
31.29 \\
31.21\end{array}$ & $\begin{array}{l}0.41 \\
0.35 \\
0.33 \\
0.41 \\
0.32 \\
0.36\end{array}$ & $\begin{array}{l}0.23 \\
0.21 \\
0.20 \\
0.21 \\
0.21 \\
0.21\end{array}$ & $\begin{array}{l}14.35 \\
14.77 \\
14.45 \\
14.55 \\
14.80 \\
14.58\end{array}$ & $\begin{array}{l}3.25 \\
3.14 \\
3.21 \\
3.23 \\
3.11 \\
3.19\end{array}$ & $\begin{array}{l}0.04 \\
0.03 \\
0.04 \\
0.06 \\
0.05 \\
0.04\end{array}$ & $\begin{array}{l}100.34 \\
100.47 \\
100.44 \\
100.93 \\
100.21 \\
100.48\end{array}$ & $\begin{array}{l}82.84 \\
83.78 \\
83.11 \\
83.07 \\
83.85 \\
83.33\end{array}$ \\
\hline $\begin{array}{l}\text { Plag } 3 \\
\text { Plag } 3 \\
\text { Plag } 3 \\
\text { Average }\end{array}$ & $\begin{array}{l}\mathrm{O} \\
\mathrm{O} \\
\mathrm{O}\end{array}$ & $\begin{array}{l}48.18 \\
48.02 \\
51.27 \\
49.16\end{array}$ & $\begin{array}{l}33.00 \\
33.04 \\
30.89 \\
32.31\end{array}$ & $\begin{array}{l}0.37 \\
0.33 \\
0.42 \\
0.37\end{array}$ & $\begin{array}{l}0.19 \\
0.21 \\
0.23 \\
0.21\end{array}$ & $\begin{array}{l}16.57 \\
16.48 \\
14.04 \\
15.70\end{array}$ & $\begin{array}{l}1.98 \\
1.95 \\
3.52 \\
2.48\end{array}$ & $\begin{array}{l}0.03 \\
0.03 \\
0.03 \\
0.03\end{array}$ & $\begin{array}{l}100.33 \\
100.05 \\
100.41 \\
100.27\end{array}$ & $\begin{array}{l}90.10 \\
90.23 \\
81.40 \\
87.24\end{array}$ \\
\hline \multicolumn{11}{|c|}{ Sample 135-834B-34R-2, 20-24 cm: } \\
\hline $\begin{array}{l}\text { Plag } 1 \\
\text { Plag } 1 \\
\text { Plag 1 } \\
\text { Plag 1 } \\
\text { Plag 1 } \\
\text { Plag 1 } \\
\text { Average }\end{array}$ & $\begin{array}{l}\mathrm{D} \\
\mathrm{D} \\
\mathrm{D} \\
\mathrm{D} \\
\mathrm{D} \\
\mathrm{D}\end{array}$ & $\begin{array}{l}46.81 \\
47.32 \\
46.79 \\
48.50 \\
47.88 \\
51.34 \\
48.11\end{array}$ & $\begin{array}{l}33.88 \\
33.58 \\
34.47 \\
32.83 \\
33.33 \\
30.88 \\
33.16\end{array}$ & $\begin{array}{l}0.31 \\
0.28 \\
0.26 \\
0.30 \\
0.36 \\
0.40 \\
0.32\end{array}$ & $\begin{array}{l}0.17 \\
0.18 \\
0.13 \\
0.19 \\
0.20 \\
0.25 \\
0.19\end{array}$ & $\begin{array}{l}17.53 \\
17.31 \\
17.81 \\
16.38 \\
16.99 \\
14.24 \\
16.71\end{array}$ & $\begin{array}{l}1.48 \\
1.75 \\
1.30 \\
2.06 \\
1.84 \\
3.62 \\
2.01\end{array}$ & $\begin{array}{c}0.01 \\
0.01 \\
0 \\
0.01 \\
0.03 \\
0.04 \\
0.02\end{array}$ & $\begin{array}{l}100.19 \\
100.43 \\
100.76 \\
100.29 \\
100.62 \\
100.76 \\
100.51\end{array}$ & $\begin{array}{l}92.85 \\
91.55 \\
93.79 \\
89.69 \\
90.97 \\
81.16 \\
90.00\end{array}$ \\
\hline $\begin{array}{l}\text { Plag } 2 \\
\text { Plag } 2 \\
\text { Plag 2 } \\
\text { Plag 2 } \\
\text { Plag 2 } \\
\text { Average }\end{array}$ & $\begin{array}{l}E \\
E \\
E \\
E \\
E\end{array}$ & $\begin{array}{l}46.91 \\
46.97 \\
46.39 \\
49.84 \\
55.89 \\
49.20\end{array}$ & $\begin{array}{l}34.01 \\
33.82 \\
33.95 \\
32.42 \\
27.84 \\
32.41\end{array}$ & $\begin{array}{l}0.41 \\
0.39 \\
0.30 \\
0.28 \\
0.71 \\
0.42\end{array}$ & $\begin{array}{l}0.17 \\
0.18 \\
0.15 \\
0.20 \\
0.10 \\
0.16\end{array}$ & $\begin{array}{l}17.78 \\
17.69 \\
17.73 \\
15.57 \\
10.47 \\
15.85\end{array}$ & $\begin{array}{l}1.45 \\
1.46 \\
1.42 \\
2.71 \\
5.43 \\
2.49\end{array}$ & $\begin{array}{l}0.03 \\
0.03 \\
0.03 \\
0.01 \\
0.06 \\
0.03\end{array}$ & $\begin{array}{r}100.75 \\
100.53 \\
99.97 \\
101.04 \\
100.50 \\
100.56\end{array}$ & $\begin{array}{l}93.02 \\
92.93 \\
93.12 \\
86.31 \\
67.88 \\
86.65\end{array}$ \\
\hline
\end{tabular}

Notes: "Mineral" is an identifier assigned within each sample; "Grain" is a global grain label assigned for the whole database to samples selected for graphic display. Oxide data in weight percent; An in molecular percent. Plag = plagioclase. 

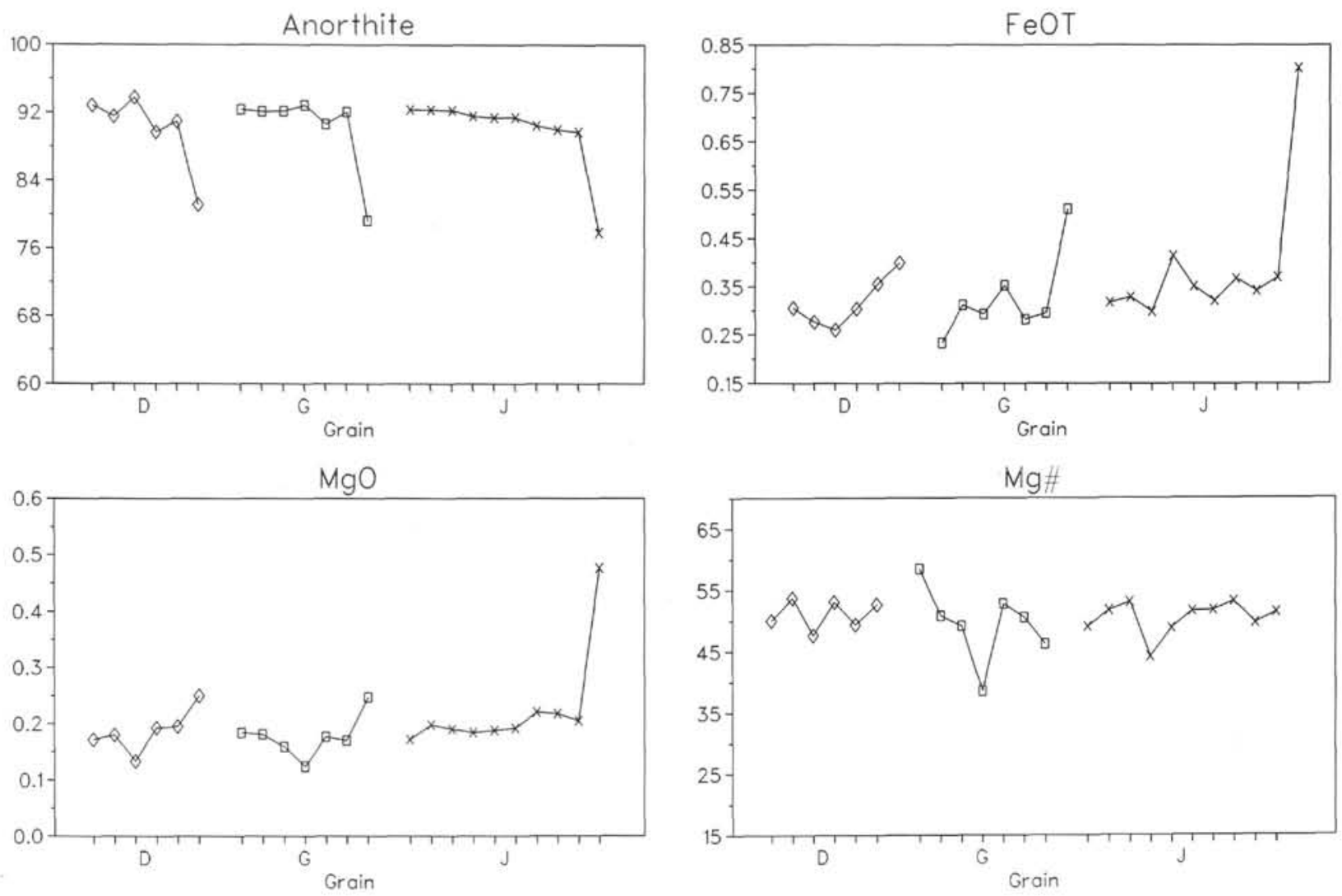

Figure 4. Variation of principal compositional parameters in selected zoned plagioclases in Hole 834B, Unit 7. Grain designations match those in Table 2. All traverses normalized to a common arbitrary scale to simplify comparison.

Table 3. Electron microprobe analyses of olivine, Hole 834B, Unit 7.

\begin{tabular}{|c|c|c|c|c|c|c|c|c|c|c|c|c|}
\hline Mineral & Grain & $\mathrm{SiO}_{2}$ & $\mathrm{TiO}_{2}$ & $\mathrm{Al}_{2} \mathrm{O}_{3}$ & $\mathrm{Cr}_{2} \mathrm{O}_{3}$ & $\mathrm{FeOT}$ & $\mathrm{MnO}$ & $\mathrm{MgO}$ & $\mathrm{CaO}$ & $\mathrm{NiO}$ & Total & Fo \\
\hline \multicolumn{13}{|c|}{ Sample $135-834 \mathrm{~B}-25 \mathrm{R}-1,28-32 \mathrm{~cm}$ : } \\
\hline $\begin{array}{l}\text { Oliv } 1 \\
\text { Oliv } 1 \\
\text { Oliv } 1 \\
\text { Oliv } 1 \\
\text { Oliv } 1 \\
\text { Oliv } 1 \\
\text { Average }\end{array}$ & $\begin{array}{l}\mathrm{S} \\
\mathrm{S} \\
\mathrm{S} \\
\mathrm{S} \\
\mathrm{S} \\
\mathrm{S}\end{array}$ & $\begin{array}{l}40.17 \\
40.37 \\
40.52 \\
40.59 \\
40.32 \\
40.34 \\
40.39\end{array}$ & $\begin{array}{l}0.07 \\
0.10 \\
0.07 \\
0.06 \\
0.07 \\
0.08 \\
0.07\end{array}$ & $\begin{array}{l}0.06 \\
0.05 \\
0.05 \\
0.03 \\
0.04 \\
0.04 \\
0.05\end{array}$ & $\begin{array}{l}0.05 \\
0.08 \\
0.05 \\
0.07 \\
0.06 \\
0.08 \\
0.06\end{array}$ & $\begin{array}{l}13.16 \\
13.08 \\
13.03 \\
13.17 \\
13.60 \\
13.76 \\
13.30\end{array}$ & $\begin{array}{l}0.13 \\
0.10 \\
0.19 \\
0.23 \\
0.18 \\
0.19 \\
0.17\end{array}$ & $\begin{array}{l}46.38 \\
46.26 \\
46.71 \\
46.10 \\
46.10 \\
45.84 \\
46.23\end{array}$ & $\begin{array}{l}0.34 \\
0.33 \\
0.34 \\
0.34 \\
0.41 \\
0.35 \\
0.35\end{array}$ & $\begin{array}{l}0.19 \\
0.20 \\
0.19 \\
0.09 \\
0.18 \\
0.17 \\
0.17\end{array}$ & $\begin{array}{l}100.56 \\
100.57 \\
101.15 \\
100.67 \\
100.96 \\
100.84 \\
100.79\end{array}$ & $\begin{array}{l}85.77 \\
85.85 \\
85.93 \\
85.60 \\
85.19 \\
85.03 \\
85.56\end{array}$ \\
\hline \multicolumn{13}{|c|}{ Sample 135-834B-25R-1, 106-110 cm: } \\
\hline $\begin{array}{l}\text { Oliv } 1 \\
\text { Oliv } 11 \\
\text { Oliv } 1 \\
\text { Oliv } 1 \\
\text { Oliv } 1 \\
\text { Oliv } 11 \\
\text { Oliv } 1 \\
\text { Average }\end{array}$ & $\begin{array}{l}\mathrm{R} \\
\mathrm{R} \\
\mathrm{R} \\
\mathrm{R} \\
\mathrm{R} \\
\mathrm{R} \\
\mathrm{R}\end{array}$ & $\begin{array}{l}40.01 \\
39.72 \\
40.27 \\
40.07 \\
40.21 \\
39.66 \\
40.19 \\
40.02\end{array}$ & $\begin{array}{l}0.06 \\
0.06 \\
0.06 \\
0.06 \\
0.06 \\
0.07 \\
0.03 \\
0.06\end{array}$ & $\begin{array}{l}0.09 \\
0.07 \\
0.06 \\
0.06 \\
0.08 \\
0.06 \\
0.07 \\
0.07\end{array}$ & $\begin{array}{l}0.09 \\
0.10 \\
0.08 \\
0.13 \\
0.13 \\
0.12 \\
0.09 \\
0.11\end{array}$ & $\begin{array}{l}12.99 \\
13.41 \\
13.28 \\
14.01 \\
13.64 \\
15.20 \\
13.14 \\
13.67\end{array}$ & $\begin{array}{l}0.25 \\
0.19 \\
0.24 \\
0.24 \\
0.13 \\
0.26 \\
0.18 \\
0.21\end{array}$ & $\begin{array}{l}46.22 \\
45.70 \\
46.22 \\
45.58 \\
45.79 \\
44.62 \\
46.16 \\
45.76\end{array}$ & $\begin{array}{l}0.34 \\
0.42 \\
0.32 \\
0.35 \\
0.34 \\
0.37 \\
0.35 \\
0.36\end{array}$ & $\begin{array}{l}0.14 \\
0.12 \\
0.11 \\
0.11 \\
0.12 \\
0.13 \\
0.10 \\
0.12\end{array}$ & $\begin{array}{r}100.19 \\
99.80 \\
100.65 \\
100.60 \\
100.51 \\
100.48 \\
100.31 \\
100.36\end{array}$ & $\begin{array}{l}85.77 \\
85.21 \\
85.53 \\
84.68 \\
85.18 \\
83.31 \\
85.67 \\
85.05\end{array}$ \\
\hline \multicolumn{13}{|c|}{ Sample 135-834B-26R-1, 73-77 cm: } \\
\hline $\begin{array}{l}\text { Oliv } 1 \\
\text { Oliv } 1 \\
\text { Oliv } 1 \\
\text { Oliv } 1 \\
\text { Oliv } 1 \\
\text { Average }\end{array}$ & $\begin{array}{l}\mathrm{Q} \\
\mathrm{Q} \\
\mathrm{Q} \\
\mathrm{Q} \\
\mathrm{Q} \\
\mathrm{Q}\end{array}$ & $\begin{array}{l}40.65 \\
40.24 \\
40.48 \\
40.35 \\
39.87 \\
40.32\end{array}$ & $\begin{array}{l}0.05 \\
0.00 \\
0.00 \\
0.00 \\
0.00 \\
0.01\end{array}$ & $\begin{array}{l}0.07 \\
0.08 \\
0.06 \\
0.04 \\
0.09 \\
0.07\end{array}$ & $\begin{array}{l}0.07 \\
0.12 \\
0.12 \\
0.12 \\
0.10 \\
0.10\end{array}$ & $\begin{array}{l}11.35 \\
11.63 \\
11.60 \\
11.61 \\
13.67 \\
11.97\end{array}$ & $\begin{array}{l}0.20 \\
0.09 \\
0.10 \\
0.07 \\
0.12 \\
0.12\end{array}$ & $\begin{array}{l}47.69 \\
47.28 \\
47.61 \\
47.72 \\
45.94 \\
47.25\end{array}$ & $\begin{array}{l}0.32 \\
0.32 \\
0.31 \\
0.30 \\
0.36 \\
0.32\end{array}$ & $\begin{array}{l}0.24 \\
0.15 \\
0.17 \\
0.14 \\
0.15 \\
0.17\end{array}$ & $\begin{array}{r}100.62 \\
99.90 \\
100.45 \\
100.36 \\
100.28 \\
100.32\end{array}$ & $\begin{array}{l}87.69 \\
87.43 \\
87.53 \\
87.59 \\
85.19 \\
87.09\end{array}$ \\
\hline \multicolumn{13}{|c|}{ Sample 135-834B-33R-2, 43-47 cm: } \\
\hline $\begin{array}{l}\text { Oliv } 1 \\
\text { Oliv } 1 \\
\text { Oliv } 1 \\
\text { Average }\end{array}$ & $\begin{array}{l}\mathrm{P} \\
\mathrm{P} \\
\mathrm{P}\end{array}$ & $\begin{array}{l}39.86 \\
40.38 \\
40.48 \\
40.24\end{array}$ & $\begin{array}{l}0.00 \\
0.00 \\
0.01 \\
0.00\end{array}$ & $\begin{array}{l}0.05 \\
0.06 \\
0.05 \\
0.05\end{array}$ & $\begin{array}{l}0.14 \\
0.13 \\
0.14 \\
0.14\end{array}$ & $\begin{array}{l}14.03 \\
11.40 \\
11.49 \\
12.31\end{array}$ & $\begin{array}{l}0.21 \\
0.20 \\
0.10 \\
0.17\end{array}$ & $\begin{array}{l}45.55 \\
47.59 \\
47.70 \\
46.95\end{array}$ & $\begin{array}{l}0.35 \\
0.33 \\
0.29 \\
0.33\end{array}$ & $\begin{array}{l}0.14 \\
0.15 \\
0.20 \\
0.16\end{array}$ & $\begin{array}{l}100.33 \\
100.23 \\
100.46 \\
100.34\end{array}$ & $\begin{array}{l}84.68 \\
87.59 \\
87.68 \\
86.65\end{array}$ \\
\hline \multicolumn{13}{|c|}{ Sample 135-834B-34R-2, 20-24 cm: } \\
\hline $\begin{array}{l}\text { Oliv } 1 \\
\text { Oliv } 1 \\
\text { Average }\end{array}$ & & $\begin{array}{l}40.49 \\
40.71 \\
40.60\end{array}$ & $\begin{array}{l}0.00 \\
0.00 \\
0.00\end{array}$ & $\begin{array}{l}0.03 \\
0.05 \\
0.04\end{array}$ & $\begin{array}{l}0.13 \\
0.14 \\
0.13\end{array}$ & $\begin{array}{l}12.43 \\
12.61 \\
12.52\end{array}$ & $\begin{array}{l}0.24 \\
0.24 \\
0.24\end{array}$ & $\begin{array}{l}46.87 \\
46.93 \\
46.90\end{array}$ & $\begin{array}{l}0.31 \\
0.34 \\
0.33\end{array}$ & $\begin{array}{l}0.21 \\
0.22 \\
0.22\end{array}$ & $\begin{array}{l}100.70 \\
101.25 \\
100.98\end{array}$ & $\begin{array}{l}86.49 \\
86.31 \\
86.40\end{array}$ \\
\hline
\end{tabular}

Notes: "Mineral" is an identifier assigned within each sample; "Grain" is a global grain label assigned for the whole database to grains selected for graphic display. All oxides in weight percent; Fo in molecular percent. Oliv = Olivine. 

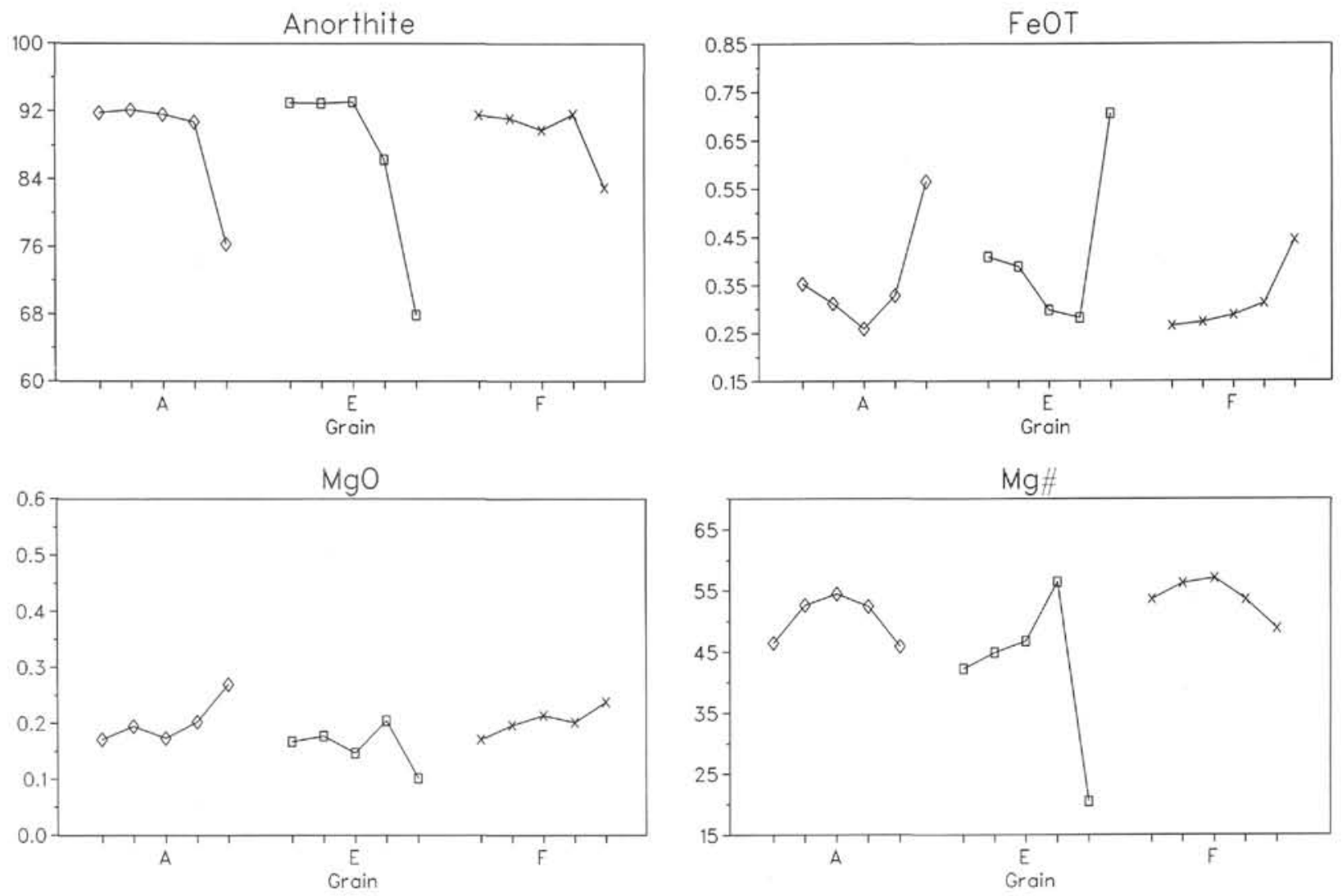

Figure 5. Variation of principal compositional parameters in selected zoned plagioclases in Hole 834B, Unit 7. Grain designations match those in Table 2. Compare with Figure 4; only the rims show significant differences. Traverse length and spacing normalized as in Figure 4.

Table 4. Electron microprobe analyses of spinel, Hole 834B, Unit 7.

\begin{tabular}{|c|c|c|c|c|c|c|c|c|c|c|c|}
\hline Mineral & Grain & $\mathrm{SiO}_{2}$ & $\mathrm{TiO} 2$ & $\mathrm{Al}_{2} \mathrm{O}_{3}$ & $\mathrm{Cr}_{2} \mathrm{O}_{3}$ & FeOT & $\mathrm{MnO}$ & $\mathrm{MgO}$ & $\mathrm{CaO}$ & $\mathrm{NiO}$ & Total \\
\hline \multicolumn{12}{|c|}{ Sample 135-834B-26R-1, 73-77 cm: } \\
\hline Spinel 1 & YY & 0.06 & 0.60 & 31.25 & 33.65 & 17.80 & 0.18 & 16.05 & 0.05 & 0.15 & 99.79 \\
\hline Spinel 1 & YY & 0.05 & 0.58 & 30.88 & 34.30 & 17.74 & 0.19 & 16.04 & 0.03 & 0.09 & 99.89 \\
\hline Spinel 1 & YY & 0.10 & 0.55 & 30.89 & 33.90 & 17.91 & 0.20 & 15.91 & 0.03 & 0.19 & 99.67 \\
\hline Spinel 1 & YY & 0.06 & 0.57 & 31.08 & 33.97 & 17.40 & 0.22 & 16.11 & 0.02 & 0.08 & 99.51 \\
\hline Average & & 0.07 & 0.58 & 31.02 & 33.95 & 17.71 & 0.20 & 16.03 & 0.03 & 0.13 & 99.72 \\
\hline
\end{tabular}

Notes: "Mineral" is an identifier assigned within each sample. "Grain" is a global grain label assigned for the whole database to samples selected for graphic display. Oxide data in weight percent.

plagioclase, and broad oscillatory-zoned outer margins that also may be sector-zoned. Another crystal (Fig. 8) shows strong oscillatory zoning from core to rim, similar to that in the second Site 841 plagioclase. Also, note the alternate development of embayments and euhedral zoning on the diagonal face toward the upper left corner of this crystal. Sector zoning is also evident between the $\{010\}$ faces (top and bottom) and the $\{001\}$ faces. A euhedral phenocryst (Plate 3 ), with internal "hopper" morphology preserved in the core, still contains patches of devitrified glass. Again, note the repetition of zoning abnormalities, which here consist of odd "tongue-like" growths toward the upper left and upper right corners of this crystal.

Plate 4, Figures A and B, shows other details. In Figure A the peculiar "jelly-roll" zoning observed in one phenocryst probably results from a fortuitous section through a region of convolute zoning. In Figure B, the ten narrow oscillatory zones at the rim are repeated around what appears to a tiny glass inclusion. This "inclusion" is more likely the end of an embayment that communicated freely with the magma in three dimensions.
Within the limitations of this study, which by no means embraces all units in the Site 834 drill holes, we have shown that much of the plagioclase does show the simpler zoning patterns more typical of MORB basalts, but local examples also exist of complex core zoning thought to be most typical of arc environments. We can only speculate at this time whether these morphologically contrasted plagioclases indicate a possible mixing event between a more MORB-like and more arc-like magma. It is otherwise difficult to understand how one set of phenocrysts could have cores that annealed and homogenized, an event that seems to require a long residence time in a stable magma chamber, whereas some other crystals escaped this event and record instability, apparently at the same period of time in terms of crystal "stratigraphy."

\section{SITE 839}

In this initial series of studies, we have examined only a few Site 839 plagioclase phenocrysts; all are from Unit 9 . These crystals display features similar to those already discussed from Sites 834 and 841. Figure $9 \mathrm{~A}$ illustrates a phenocryst that displays alternating chaotic and euhedral growth. The chaotic zone near the center of the crystal evolves toward euhedral growth through an intermediate region of concentric lobes separated by periodic dislocations, similar to those described at Site 841. Not all crystals show this complexity; those in Figure 9B are part of a monomineralic clot of plagioclase in which the oscillatory core zones appear to have been partly annealed and homogenized; they are similar to the annealed cores described at Site 834. Plate 5 shows another phenocryst that again reveals alternating periods of chaotic and euhedral growth, with an embayment confined to one face. Note also the apparent unconformities between the embayment "fill" and the euhedral faces approaching from either side. These may not be true erosional (or dissolution) unconformities, but may simply mark much more rapid growth within the embayment relative to the outer faces. 


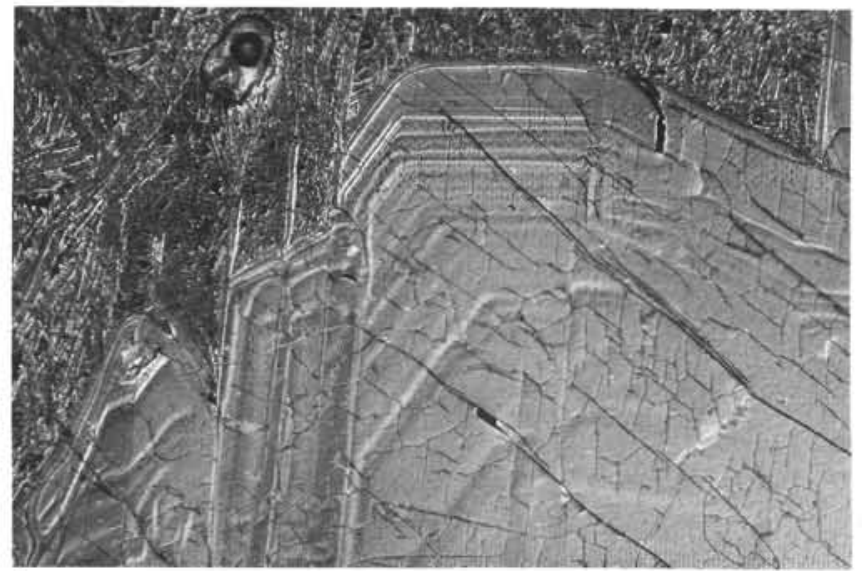

A

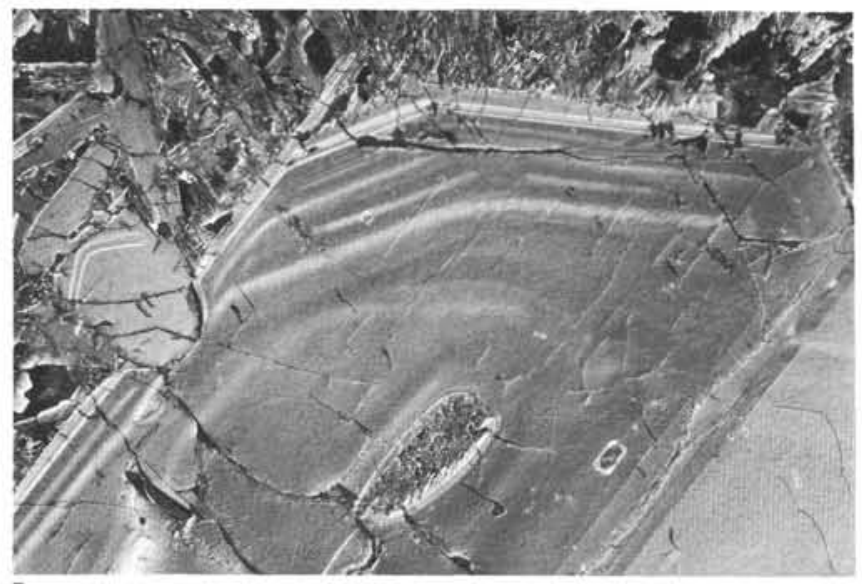

B

Figure 6. Comparison of zoning in plagioclase from the upper and lower parts of Hole 834B, Unit 7. A. Sample 135-834B-24R-1, 28-32 cm, illustrating subdued central core zoning and sharper marginal oscillatory zoning. B. Sample 135-834B-33R-2, 43-47 cm, illustrating faint core zoning around a large ovoid area of possible devitrified glass, and sharper oscillatory zoning along the outer margin. Note that both crystals have delicate "spikes" of plagioclase attached to their outermost margins, both associated with the final quenching event. Width of field in both photos is about $400 \mu \mathrm{m}$.

We conclude that the Site 839 Unit 9 plagioclase displays a variety of zoning morphologies, which again indicate that not all crystals have evolved in identical environments. Other than mixing, one possible explanation for this is that water content in the melt has been variable and distributed inhomogeneously throughout the magma during the growth of these crystals. A similar explanation has been suggested by Gaetani et al (this volume) to account for the variable petrographic evidence for the olivine-hypersthene reaction in Site 839 Unit 1 rocks. Both plagioclase composition (and stability) and the "olivine-out" reaction are sensitive to water content and pressure, and both could vary significantly in, for example, a long dike-like or pipe-like magma chamber or conduit system. In any event, the zoning variations we have observed argue against magmatic evolution within a simple, closed, stable, homogeneous chamber at a fixed depth.

\section{CONCLUSIONS}

Plagioclase phenocrysts at Sites 841 and 839 display the complex repetitive and locally chaotic zoning similar to that observed in lavas from other circum-Pacific volcanoes. This certainly is consistent with the geochemical indications of an "arc signature" in these rocks. Site

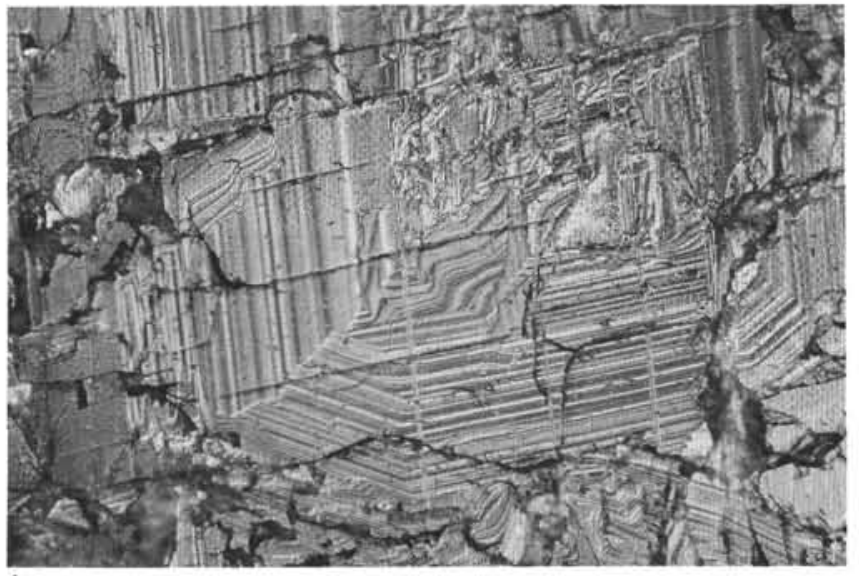

A

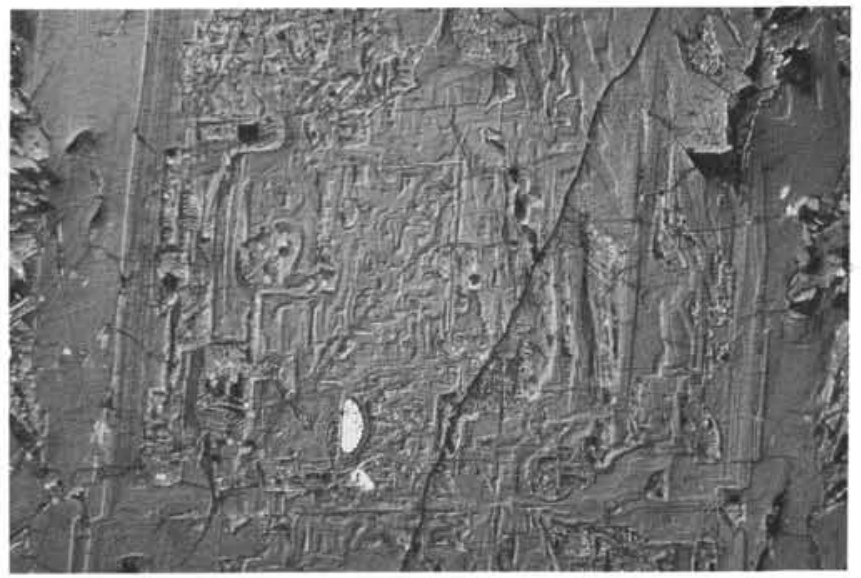

B

Figure 7. Hieroglyphic zoning in two crystals from the middle portion of Hole 834B, Unit 7. A. Sample 135-834B-31R-3, 53-57 cm; note apparent sectorzone boundary crossing the broad outer oscillatory zone toward the lower left corner of the crystal. B. Sample 135-834B-26R-1, 73-77 cm; detail of central core of phenocryst to show the complex detail in the hieroglyphic pattern. Width of field in both photos is about $200 \mu \mathrm{m}$.

834 phenocrysts most commonly show much simpler zoning patterns, with a narrow sodic rim on a broad, almost homogeneous core. But the presence of scattered phenocrysts with more complex internal zoning patterns indicates subtle complications in the evolution of Site 834 lavas. These phenocrysts show arc-like complexities, including excellent examples of "hieroglyphic" core zoning. These may indicate mixing of the more "MORB-like" Unit 7 magma with a small amount of arc-derived magma, a not unreasonable possibility given the proximity to the old Lau Ridge arc. These crystals also could have been derived as contaminants from an older, arc-related lava later intruded by the Unit 7 magma. It is obvious that our limited study has revealed the probability of substantial complexity in magmatic evolutionary history at these sites. Much more detailed work will be required to clarify the details of this history.

\section{ACKNOWLEDGMENTS}

This work was supported in part by a grant from USSAC to W.B. Bryan, and a NSERC operating grant to T.H. Pearce. We thank Glenn Gaetani for assistance with the microprobe analyses, and Kristin Nilsson for advance access to glass analyses from Hole 834B, Unit 7. Comments by A. Ewart and L. Forsythe during the post-cruise meeting also contributed to some of the ideas presented here. 


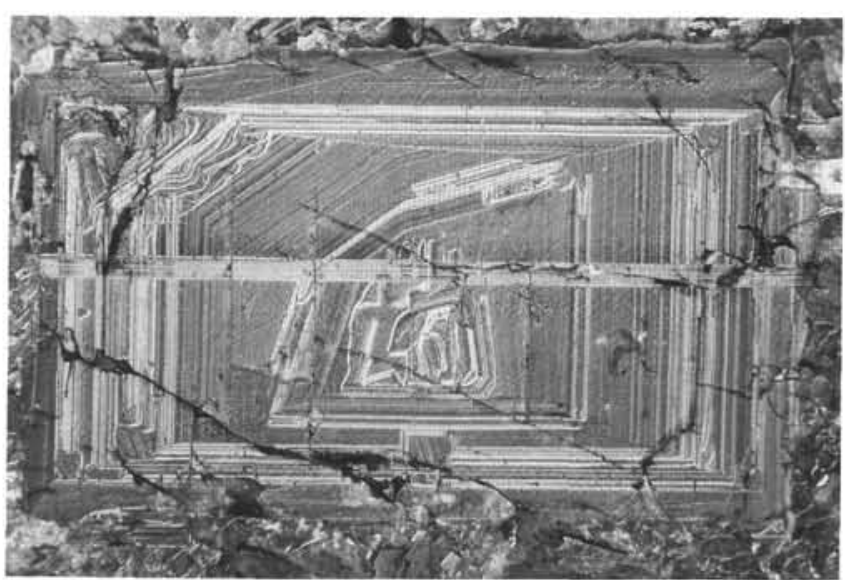

Figure 8. Core-to-rim oscillatory zoning in a euhedral plagioclase phenocryst from Hole 834B, Unit 7 (Sample 135-834B-31R-3, 53-57 cm). Width of field is about $200 \mu \mathrm{m}$. See text for discussion.

\section{REFERENCES $*$}

Anderson, A.T., Jr., 1983. Oscillatory zoning of plagioclase: Nomarski interference contrast microscopy of etched sections. Am. Mineral., 68:125-129.

Blackerby, B.A., 1968. Convolute zoning of plagioclase phenocrysts in Miocene volcanics from the western Santa Monica Mountains, California. Am. Mineral., 53:954-962.

Parson, L., Hawkins, J., Allan, J., et al., 1992. Proc. ODP, Init. Repts., 135 (Pt. 2): College Station, TX (Ocean Drilling Program).

Pearce, T.H., 1984. Optical dispersion and zoning in magmatic plagioclase: laser interference observations. Can. Mineral., 22:383-390.

Pearce, T.H., and Kolisnik, A.M., 1990. Observations of plagioclase zoning using interference imaging. Earth-Sci. Rev, 29:9-26.

Tsuchiyama, A., 1985. Dissolution kinetics of plagioclase in the melt of the system diopside-albite-anothite, and origin of dusty plagioclase in andesites. Contrib. Mineral. Petrol., 89:1-16.

Wiebe, R.A., 1968. Plagioclase stratigraphy: a record of magmatic conditions and events in a granitic stock. Am. J. Sci., 266:690-703.

\footnotetext{
* Abbreviations for names of organizations and publication titles in ODP reference lists follow the style given in Chemical Abstracts Service Source Index (published by American Chemical Society).
}

Date of initial receipt: 6 July 1992

Date of acceptance: 11 February 1993

Ms 135SR-132

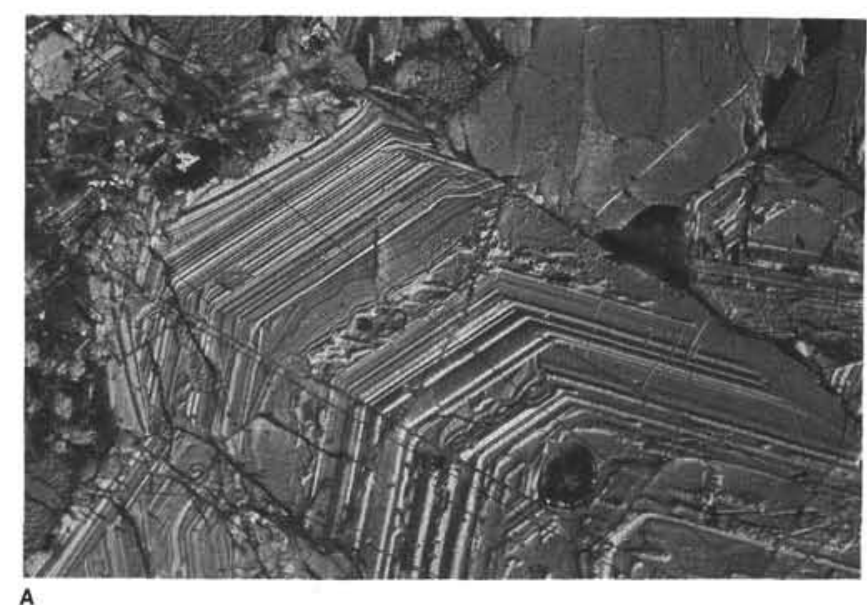

A

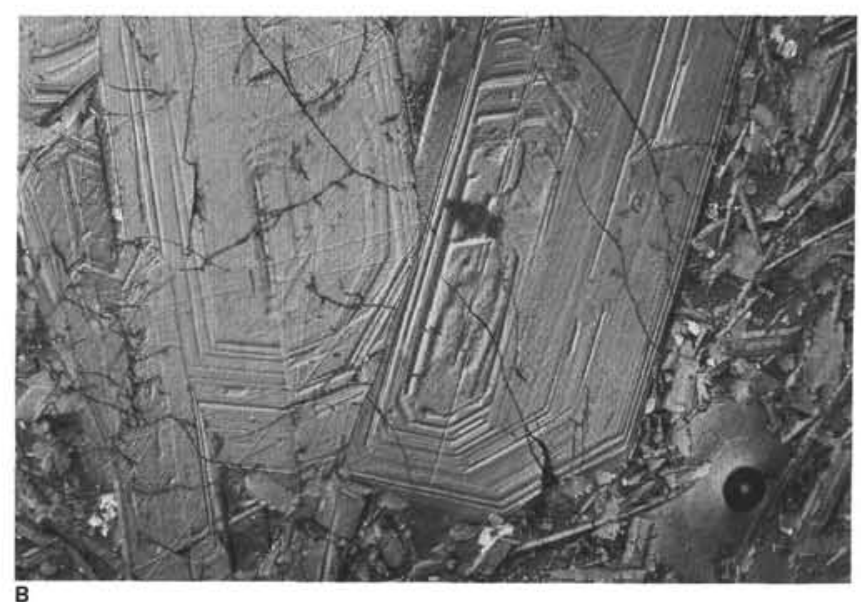

Figure 9. Reflected light NDIC images of zoning in plagioclase from an andesite in Hole 839B, Unit 9. A. Sample 135-839B-30R-1, 23-27 cm; very fine scale $(2-3 \mu \mathrm{m})$ planar zoning alternates with complex nonplanar zoning. The subdued appearance of the core zones suggests that it is much older than the rim and that diffusion has had time to obscure the sharp contacts between zones. Width of field is about $150 \mu \mathrm{m}$. B. Sample 135-839B-29R-1, 70-77 cm; detail of an intergrowth of two microphenocrysts of plagioclase in a crystal clot. This clot is monomineralic; only plagioclase is present. Width of field is about $200 \mu \mathrm{m}$. 

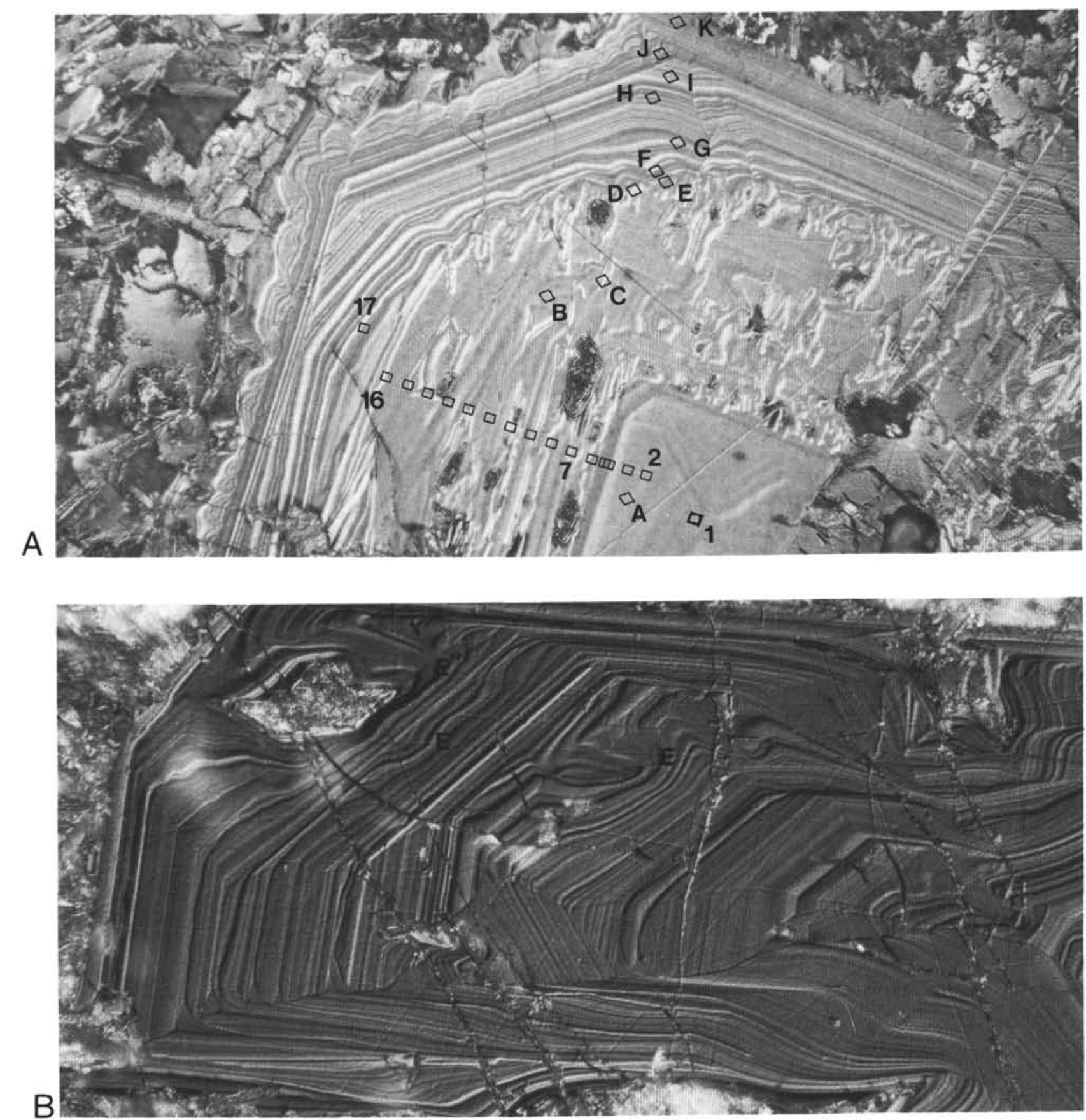

Plate 1. Reflected light Nomarski differential interference contrast (NDIC) image in reflected light of plagioclase phenocrysts in upper Miocene basaltic andesite sills. A. Sample 135-841B-18R-2, 14-18 cm, illustrates calcic core $\left(\mathrm{An}_{92}\right)$, reaction zone in the mantle and oscillatory rim zoned to $<\mathrm{An}_{75}$. Probe traverses 1 (diamonds) and 2 (squares) are indicated. Width of field about $300 \mu \mathrm{m}$. B. Sample 135-841B-25R-4, 10-14 cm, illustrates high-power reflected NDIC image of part of a phenocryst. Repetitive convolute zoning (E, E', $E^{\prime \prime}$ ) is well developed in this sample; see text for discussion. Width of field is about $150 \mu \mathrm{m}$. 


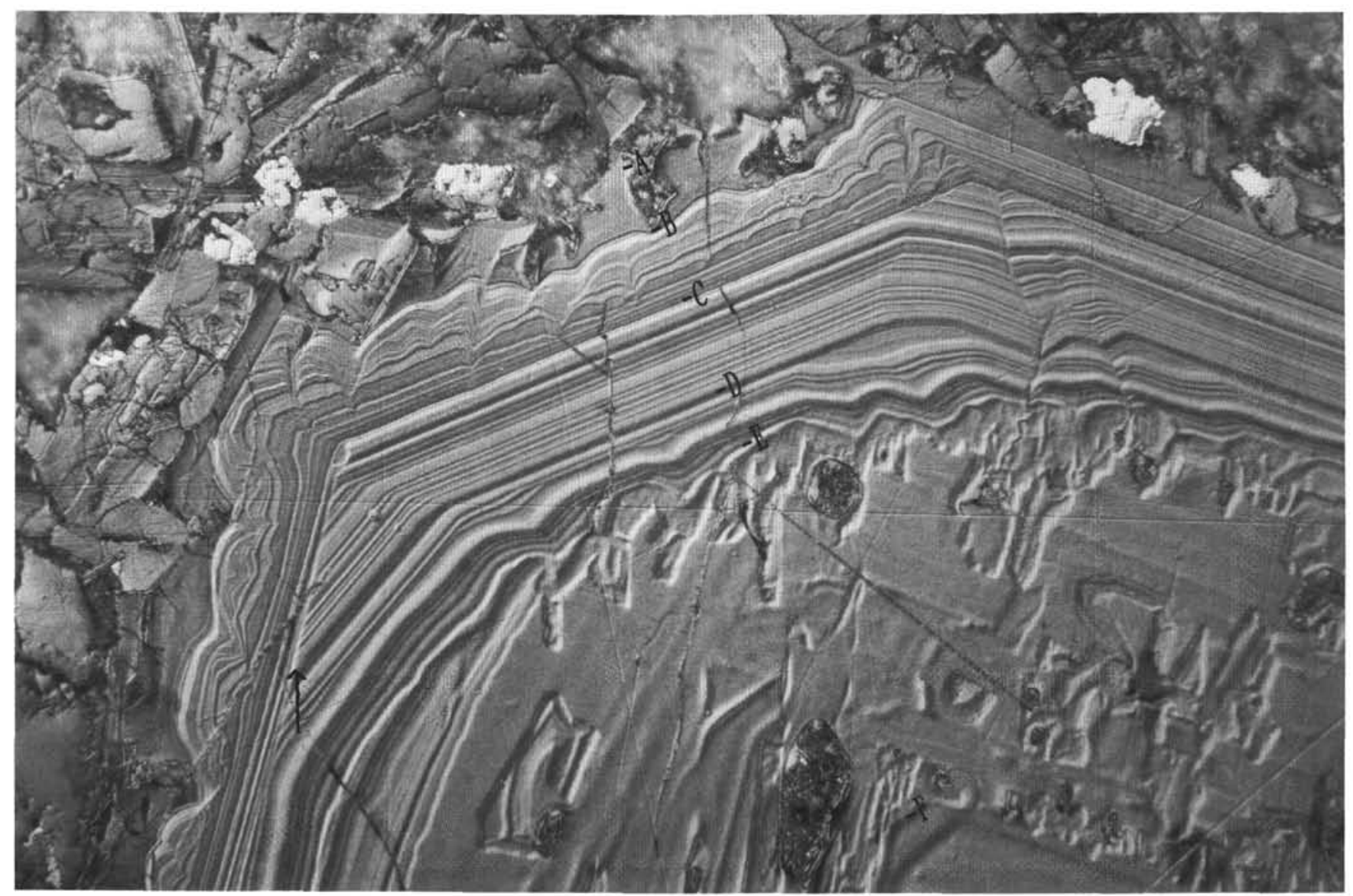

Plate 2. Reflected light NDIC image of part of the crystal in Plate 1, Figure A, from Hole 841B. Details of the cross-cutting dislocations within the reaction zone are clearly visible. Arrow indicates possible sector zone boundary. See text for detailed stratigraphic interpretation. Width of view is about $200 \mu \mathrm{m}$. 


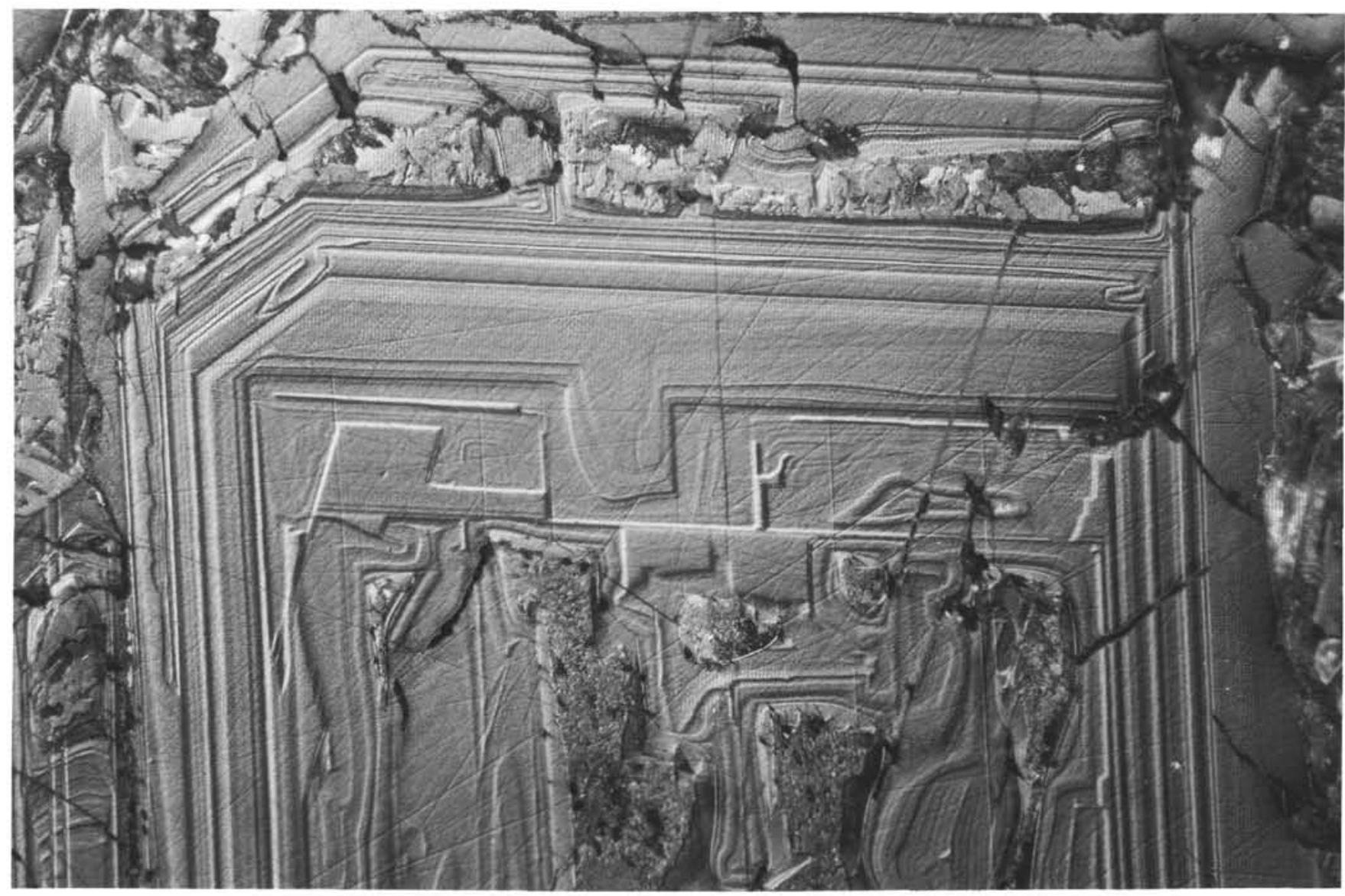

Plate 3. Reflected light NDIC image of a plagioclase from Hole 834B, Unit 7 (Sample 135-834B-33R-2, 43-47 cm). Hopper-style growth appears to have taken place several times during growth of this crystal. See text for more details. Width of view is about $200 \mu \mathrm{m}$. 


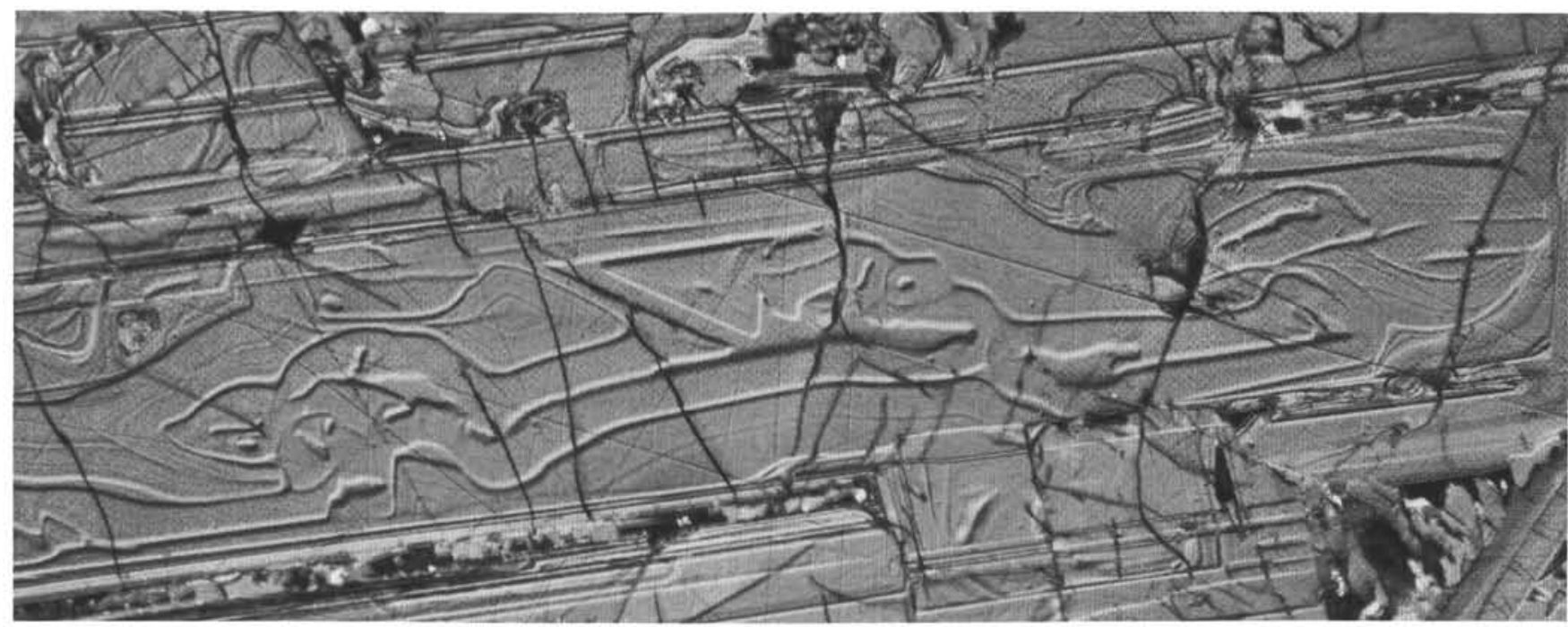

A

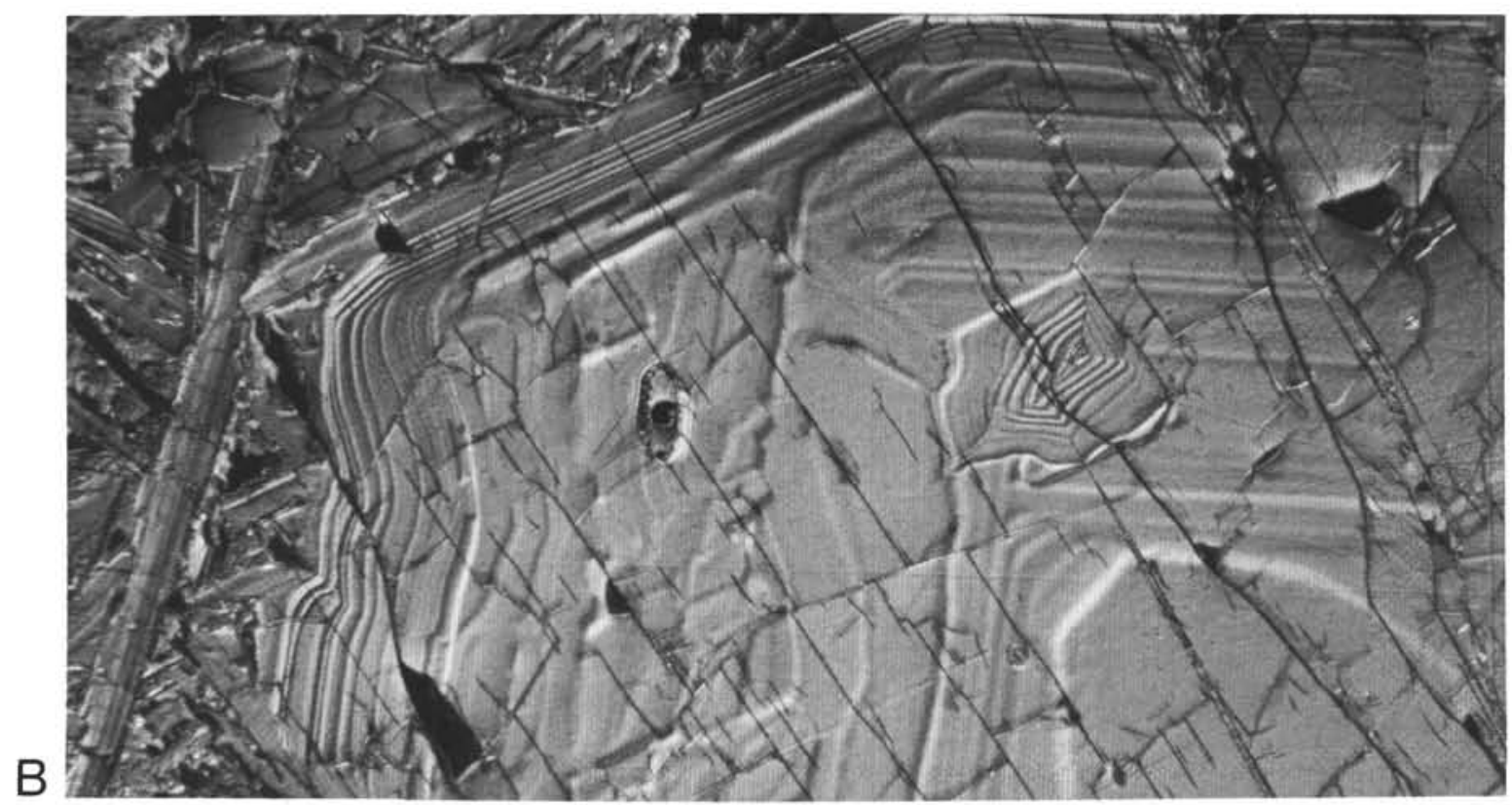

Plate 4. Zoning patterns in basalt, Sample 135-834B-26R-1, 73-77 cm, from Hole 834B, Unit 7. A. "Jelly-roll" texture, possibly a result of shallow intersection of the plane of section and a zone of convolute zoning. Width of view is about $150 \mu \mathrm{m}$. B. Duplication of zoning pattern of ten zones at the rim and in a small inlier. Width of view is about $350 \mu \mathrm{m}$. 


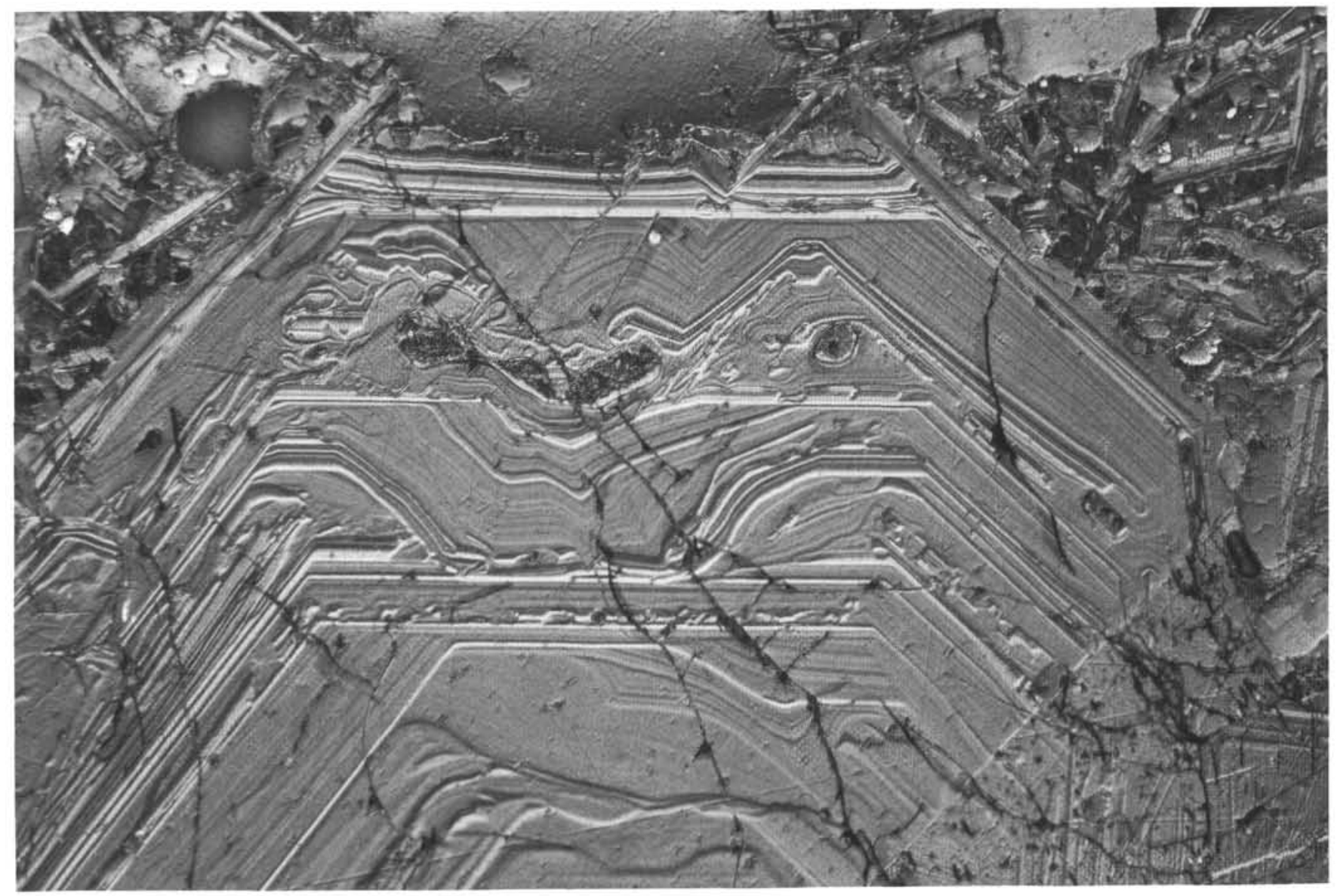

Plate 5. Reflected light NDIC image of one end of a plagioclase phenocryst in an andesite from Hole 839B, Unit 9 (Sample 135-839B-29R-1, 70-77 cm). Note the delta-like fill in the embayments, and the more subtle, delta-like stratigraphy in the oscillatory zone in the upper right. See text for further discussion. Width of field is about $200 \mu \mathrm{m}$. 